\title{
Multi-Frequency Control of Faraday Wave Patterns
}

\author{
Chad M. Topaz \\ Department of Mathematics, UCLA, Los Angeles, California 90095, USA * \\ Jeff Porter \\ Department of Applied Mathematics, University of Leeds, Leeds LS2 9JT, UK \\ Mary Silber \\ Department of Engineering Sciences and Applied Mathematics, \\ Northwestern University, Evanston, Illinois 60208, USA
}

(Dated: October 23, 2018)

\begin{abstract}
We show how pattern formation in Faraday waves may be manipulated by varying the harmonic content of the periodic forcing function. Our approach relies on the crucial influence of resonant triad interactions coupling pairs of critical standing wave modes with damped, spatio-temporally resonant modes. Under the assumption of weak damping and forcing, we perform a symmetrybased analysis that reveals the damped modes most relevant for pattern selection, and how the strength of the corresponding triad interactions depends on the forcing frequencies, amplitudes, and phases. In many cases, the further assumption of Hamiltonian structure in the inviscid limit determines whether the given triad interaction has an enhancing or suppressing effect on related patterns. Surprisingly, even for forcing functions with arbitrarily many frequency components, there are at most five frequencies that affect each of the important triad interactions at leading order. The relative phases of those forcing components play a key role, sometimes making the difference between an enhancing and suppressing effect. In numerical examples, we examine the validity of our results for larger values of the damping and forcing. Finally, we apply our findings to onedimensional periodic patterns obtained with impulsive forcing and to two-dimensional superlattice patterns and quasipatterns obtained with multi-frequency forcing.
\end{abstract}

PACS numbers: 05.45.-a, 47.35.+i, 47.54.+r, 89.75.Kd

\section{INTRODUCTION}

Parametrically forced surface waves have proven to be a rich and versatile source of patterns since their initial observation by Michael Faraday in 1831 [1]. These Faraday wave patterns are composed of standing waves set up in response to periodic vertical vibration of sufficient strength. Early investigations (see 2, 3] for reviews) used a sinusoidal forcing function and focused on simple patterns such as stripes, squares, and hexagons, which oscillate in subharmonic response to the forcing. Recently, experimentalists have used multi-frequency forcing to generate more complex states such as quasipatterns and superlattice patterns [4, 5, 6, 7, 8, 9, 10, 11]. These observations have, in turn, fueled theoretical interest in such patterns and in multi-frequency forcing 12, 13, 14, 15, 16, 17, 18, 19.

The use of multi-frequency forcing requires the selection of a large number of control parameters. The forcing frequencies, their amplitudes, and their relative phases may all affect the pattern formation problem in a nontrivial way [4, 12, 14, 20]. Further complexity arises from the presence of multiple length scales. In addition to the length scales driven by the various forc-

*Electronic address: topaz@ucla.edu ing frequencies in accordance with the parametric (i.e., subharmonic) resonance conditions, there are numerous damped modes that are driven nonlinearly. Many of these can significantly influence the dynamics of the critical modes. For instance, in the case of two-frequency forcing, the damped mode that oscillates at the so-called difference frequency is important for selecting superlattice patterns [19].

Resonant triad interactions - the lowest order nonlinear interactions - provide a useful framework for investigating the relationship between the many control parameters and length scales in the multi-frequency forced Faraday wave problem. Resonant triads that couple two critical modes with a damped, spatiotemporally resonant mode play a key role in the nonlinear pattern selection process. Most of these damped modes function as energy sinks, effectively creating an anti-selection mechanism that suppresses the triad interaction and thereby favors patterns which avoid the corresponding resonant angle. However, other damped modes act as energy sources, providing a positive selection mechanism that helps stabilize patterns involving the associated resonant angle. The effect of different damped modes on pattern selection is investigated in 20], which, for forcing functions with up to three frequency components, determines the most important damped modes, their effect (enhancing or suppressing) on associated patterns, and the dependence of the nonlinear interaction on the forcing frequencies, amplitudes, and relative phases. These results are used to 
interpret recent Faraday wave experiments that produced complex patterns, namely, a two-frequency forced superlattice pattern in 7] and a three-frequency forced quasipattern in [11]. The approach developed in [20] follows from a systematic consideration of weakly broken symmetries: time translation, time reversal, and Hamiltonian structure (see [12]), and is therefore most relevant for systems with weak damping and forcing. In this limit the vastness of the control parameter space can be an asset, enabling one to enhance or suppress particular triad interactions simply by tuning the appropriate forcing parameters.

In this paper, we adopt the same prescriptive approach to Faraday wave pattern formation, describing in more detail the technique for exploiting weakly broken symmetries, and extending the results of [20] to forcing functions containing arbitrarily many Fourier components. We determine which damped modes are favored by a strong nonlinear coupling and tabulate how the corresponding resonant triad interactions depend on the forcing parameters. A somewhat surprising result, which makes this project feasible, is that for a given damped mode there are at most five out of the potentially infinite number of forcing frequency components in the forcing function that affect the resonant triad interaction at leading order in the damping parameter $\gamma$ (defined below). We investigate numerically the validity of our predictions with respect to the small $\gamma$ assumption. This is important for understanding the extent to which the symmetry-based picture we develop here can be applied to realistic experiments. We then use several different numerical examples to illustrate how the resonant triad interactions most relevant to pattern formation may be controlled through a judicious choice of forcing parameters.

The remainder of this paper is organized as follows. In Section [II we review basic ideas concerning the importance of resonant triad interactions to Faraday wave pattern formation, including a discussion of some of the previous theoretical and experimental work. Section [11]contains our symmetry-based analysis. We enumerate the most important weakly damped modes, calculate their effect on pattern formation, and determine the dependence of this effect on the forcing parameters. Section IV contains a general discussion of the symmetry-based results. We study their range of validity with respect to $\gamma$ by comparing the symmetry-based predictions to numerical results obtained using the Zhang-Viñals Faraday wave equations [21]. In Section $\nabla$ we apply our symmetrybased results in several examples. In the first application, we consider weakly-nonlinear periodic patterns forced by a repeated sequence of $\delta$-functions of alternating sign. In accordance with the results first reported in [22], we demonstrate how, by varying the spacing between the pulses, we may control the amplitude of the pattern. In the second application, we show how to construct a fivefrequency forcing function which leads to dramatic stabilization of a complex pattern, namely an SL-I superlattice pattern of the type observed in [7]. In the third example, we conjecture about a seven-frequency forcing function which should be conducive to the experimental observation (as yet, lacking) of 14-fold quasipatterns. We summarize and conclude in Section DI

\section{BACKGROUND}

We lay the groundwork for our new results by reprising basic ideas from [12, 17, 19, 20, 23, 24] on the role of resonant triads in Faraday wave pattern formation. We consider Faraday waves on an unbounded horizontal domain subjected to an arbitrary periodic forcing function $f(t)$. We use a dimensionless time $\tau$ such that the common frequency is one, and expand $f(\tau)$ in a Fourier series:

$$
f(\tau)=\sum_{u \in \mathbb{Z}^{+}} f_{u} e^{i u \tau}+c . c ., \quad f_{u} \in \mathbb{C}
$$

where $u=m, n, p, \ldots$ are the forcing frequencies (distinct and co-prime), $\left|f_{u}\right|$ are the forcing amplitudes, and $\phi_{u}=\arg \left(f_{u}\right)$ are the corresponding phases. Without loss of generality, we take $m$ to be the "dominant" frequency, i.e. we assume that $f_{m}$ (to lowest order) is the component that drives the critical modes (this does not necessarily mean that $\left|f_{m}\right|$ is the largest of the $\left|f_{u}\right|$ ). There exists a bifurcation point $\left|f_{m}\right|=\left|f_{m}\right|^{\text {rrit }}$ which depends on the physical properties of the fluid, and on the other $f_{u}$, below which the flat fluid state is stable to perturbations of all wave numbers, and at which perturbations of (generically) one critical wave number $k_{c}$ become neutrally stable. We consider the properties of resonant triads in a vicinity of this bifurcation in parameter space.

Three wave, or triad, resonance is the simplest nonlinear mechanism by which different waves may interact. The three waves involved have Fourier wave vectors $\mathbf{k}_{j}$, $j=1,2,3$, satisfying

$$
\mathbf{k}_{1}+\mathbf{k}_{2}=\mathbf{k}_{3} .
$$

In this paper we are interested in the influence of the damped modes that are driven nonlinearly (through resonant triad interaction) by the critical modes. Hence two of the wave vectors have the critical value $\left|\mathbf{k}_{1}\right|=$ $\left|\mathbf{k}_{2}\right|=k_{c}$. These waves, to first approximation, respond subharmonically to the dominant forcing component $m$ and thus oscillate with predominant frequency $m / 2$. The third wave in the triad has wave number $\left|\mathbf{k}_{3}\right|=k_{d}$ and is associated with a damped mode with dominant frequency $\Omega$. The values of $\Omega$ most relevant to Faraday wave pattern formation are determined in Section III The condition (2) defines an angle of spatial resonance $\theta_{\text {res }} \in[0, \pi)$ between the two critical modes:

$$
\cos \frac{\theta_{\mathrm{res}}}{2}=\frac{k_{d}}{2 k_{c}} .
$$

We exclude the cases $\theta_{\text {res }}=\pi / 3$ and $\theta_{\text {res }}=2 \pi / 3$ since these correspond to hexagons and $k_{d}$ would then not be 
damped. Figure 1 shows Fourier space diagrams corresponding to the resonant triad described above.
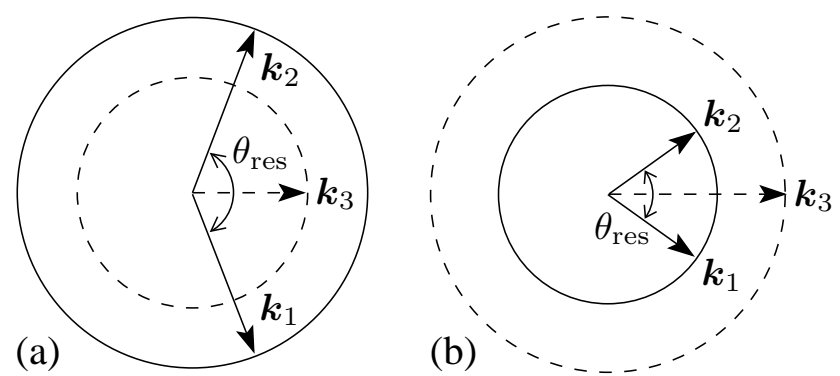

FIG. 1: Fourier space diagram of spatially resonant triads satisfying (2). The two neutrally stable modes have wave number $\left|\mathbf{k}_{1}\right|=\left|\mathbf{k}_{2}\right|=k_{c}$ and oscillate with dominant frequency $m / 2$. The damped mode has $\left|\mathbf{k}_{3}\right|=k_{d}$ and oscillates with dominant frequency $\Omega$. (a) $k_{d}<k_{c}$. (b) $k_{c}<k_{d}<2 k_{c}$.

In the presence of damping, the primary Faraday instability leads to standing waves (SW). We associate with the wave vectors $\mathbf{k}_{j}$ three complex amplitudes $A_{j}$ which describe the slow-time evolution of the three standing wave modes pictured in Figure 1 (the fast-time subharmonic oscillation of the waves has been factored out; see, e.g., 17]). Evolution equations for the $A_{j}$ can be obtained by applying a standard reduction procedure to the governing equations, as we shall do in Section $\nabla$. However, at this point we are concerned only with the form of these equations, which is determined by the spatial symmetries. The action of spatial translation is

$$
\begin{gathered}
T_{\Theta}: A_{j} \rightarrow A_{j} e^{i \theta_{j}}, \\
\Theta=\left(\theta_{1}, \theta_{2}\right), \quad \theta_{1}, \theta_{2} \in[0,2 \pi), \quad \theta_{3}=\theta_{1}+\theta_{2},
\end{gathered}
$$

while a reflection about $\mathbf{k}_{3}$ leads to

$$
\kappa: A_{1} \leftrightarrow A_{2},
$$

and a rotation by $\pi$ acts as

$$
\mathcal{R}: A_{j} \rightarrow \bar{A}_{j}
$$

Equivariance under these three symmetries (see, e.g., [25]) requires that the differential equations describing the dynamics of the $A_{j}$ take the form

$$
\begin{aligned}
\dot{A_{1}=} & \Lambda_{1} A_{1}+\alpha_{1} \bar{A}_{2} A_{3} \\
& +\left(a\left|A_{1}\right|^{2}+b_{0}\left|A_{2}\right|^{2}+b_{1}\left|A_{3}\right|^{2}\right) A_{1}, \\
\dot{A_{2}=} & \Lambda_{1} A_{2}+\alpha_{1} \bar{A}_{1} A_{3} \\
& +\left(a\left|A_{2}\right|^{2}+b_{0}\left|A_{1}\right|^{2}+b_{1}\left|A_{3}\right|^{2}\right) A_{1}, \\
\dot{A_{3}=} & \Lambda_{2} A_{3}+\alpha_{2} A_{1} A_{2} \\
& +\left(b_{2}\left|A_{1}\right|^{2}+b_{2}\left|A_{2}\right|^{2}+b_{3}\left|A_{3}\right|^{2}\right) A_{3},
\end{aligned}
$$

to cubic order. The dot represents differentiation with respect to a slow time scale. All coefficients are real.

Because $A_{1}$ and $A_{2}$ are neutrally stable modes and $A_{3}$ is linearly damped (i.e., $\Lambda_{1}=0$ and $\Lambda_{2}<0$ ), a center manifold reduction can be used to eliminate $A_{3}$. We find

$$
A_{3}=-\frac{\alpha_{2}}{\Lambda_{2}} A_{1} A_{2}+\ldots,
$$

in a neighborhood of the origin. The (unfolded) bifurcation problem, to cubic order, becomes

$$
\begin{aligned}
& \dot{A}_{1}=\Lambda_{1} A_{1}+a\left|A_{1}\right|^{2} A_{1}+b\left(\theta_{\text {res }}\right)\left|A_{2}\right|^{2} A_{1}, \\
& \dot{A}_{2}=\Lambda_{1} A_{2}+a\left|A_{2}\right|^{2} A_{2}+b\left(\theta_{\text {res }}\right)\left|A_{1}\right|^{2} A_{2},
\end{aligned}
$$

where

$$
b\left(\theta_{\text {res }}\right)=b_{0}+b_{\text {res }}, \quad b_{\text {res }}=-\frac{\alpha_{1} \alpha_{2}}{\Lambda_{2}} .
$$

The coefficient $b(\theta)$ is the cross-coupling coefficient for SW oriented at an angle $\theta$ relative to each other and, above, it is evaluated at the angle of spatial resonance $\theta=\theta_{\text {res }}$. The resonant contribution $b_{\text {res }}$ arises from the presence of the damped $k_{d}$ mode.

The resonant angle $\theta_{\text {res }}$ ranges from 0 to $\pi$ as $k_{d}$ varies from $2 k_{c}$ to 0 . When $k_{d}$ is such that the natural frequency $\Omega\left(k_{d}\right)$ of the damped mode equals (or is nearly equal to) one of the special values that promotes a strong nonlinear coupling (as determined in Section III) the contribution $b_{\text {res }}$ to $b\left(\theta_{\text {res }}\right)$ can be significant. This typically happens when $\alpha_{1}$ and $\alpha_{2}$ become large in magnitude, and/or when $\Lambda_{2}$ becomes small in magnitude. The resonant contribution will then have a major effect on the stability of associated patterns.

Consider further the system (9) which has as steadystate solutions the trivial state $\left|A_{1}\right|=\left|A_{2}\right|=0$, the symmetry-related "striped" states $\left|A_{1}\right|>0,\left|A_{2}\right|=0$ and $\left|A_{2}\right|>0,\left|A_{1}\right|=0$, and the "rhombic" mixed-mode solution $\left|A_{1}\right|=\left|A_{2}\right|$. We assume that $a<0$, so that the bifurcation to the striped state is supercritical. A straightforward analysis yields the following stability results summarized by Figure 2 For $b$ sufficiently negative, i.e., $b=b_{0}+b_{\text {res }}<a$, the (supercritical) branch of rhombic states with angle $\theta_{\text {res }}$ is unstable. If $b$ is increased such that $|b|<|a|$ (typically due to $b_{\text {res }}>0$ balancing $b_{0}<0$ ) then the two modes mutually enhance each other's growth, and the rhombic pattern is stable. If $b$ is increased further (due to an even larger, positive $b_{\text {res }}$ ) such that $b>-a$, then the rhombic state bifurcates subcritically. However, with the addition of fifth order terms (or higher) it is possible, even likely, that for the subcritical case, the unstable mixed-mode branch turns around at a saddle-node bifurcation and creates a branch of stable, finite amplitude rhombic states. Thus, we do not want to be unduly limited by the form of (9). In the initial stages of the pattern selection process, when modes on the critical circle are beginning to grow and compete, there will surely be an advantage for combinations that mutually enhance each other's growth. For these reasons we say that triad interactions contributing $b_{\text {res }}>0$ are enhancing and those giving $b_{\text {res }}<0$ are suppressing.

The above example is just one very basic instance of the importance of resonant triads. In fact, triad resonances have implications far beyond the (in)stability of 

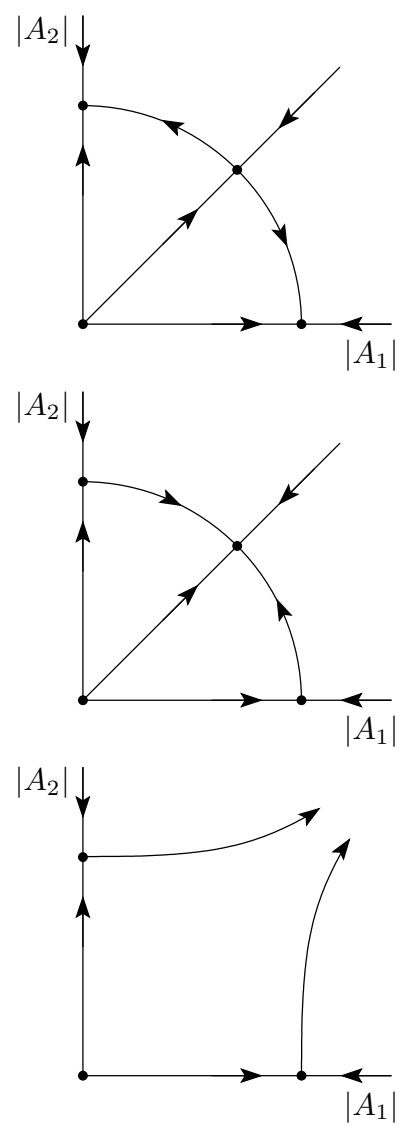

FIG. 2: Three qualitatively different phase portraits corresponding to (9) with $a<0, \Lambda_{1}>0$. Top: $b<a$. Middle: $a<b<-a$. Bottom: $b>-a$.

rhombic patterns. They may affect the stability of patterns within the framework of any Faraday wave bifurcation problem possessing a subspace with dynamics described by (9); see, for instance, 14, 17, 19, 20, 21, 23]. In these cases, the logic is the same: $b_{\text {res }}>0$ enhances patterns involving the resonant angle $\theta_{\text {res }}$ while $b_{\text {res }}<0$ suppresses them.

The triad interactions discussed in this paper have implications for one-dimensional waves as well. In this case, with $\mathbf{k}_{1}=\mathbf{k}_{2}$, the resonance condition (2) becomes simply

$$
2 k_{c}=k_{d},
$$

which is the familiar $1: 2$ spatial interaction. When the natural frequencies of the two waves are such that a strong nonlinear coupling is allowed (as we detail in Section III) we expect additional contributions to the cubic self-interaction coefficient $a$ in the SW equation

$$
\frac{d A_{1}}{d T}=\Lambda_{1} A_{1}+a\left|A_{1}\right|^{2} A_{1},
$$

which is simply (9) restricted to one spatial dimension. Since there is no spatial angle $\theta$ to tune, we may arrange for a resonant situation (11) by varying parameters in the dispersion relation, as in [19, 23].

\section{SYMMETRY CALCULATIONS}

We use the approach developed in [12, 20, 24] to determine how the resonant contribution $b_{\text {res }}$ to the cross coupling coefficient $b\left(\theta_{\text {res }}\right)$ in (9) depends on the forcing function (11). We consider a system of six traveling wave (TW) modes (see also [19]) having the same wave vectors $\mathbf{k}_{j}$ as the three SW modes described in Section II It is advantageous to consider TW first because the action of the temporal symmetries on $\mathrm{TW}$ is simple while on SW it is not. In this way, we make full use of the temporal symmetry and Hamiltonian structure before reducing the TW equations to the desired SW equations by means of a center manifold reduction. We thus expand the fluid surface height $h(\mathbf{x}, t), \mathbf{x} \in \mathbb{R}^{2}$ as

$$
h(\mathbf{x}, t)=\sum_{j=1}^{3} \sum_{ \pm} Z_{j}^{ \pm}(t) e^{i\left(\mathbf{k}_{j} \cdot \mathbf{x} \pm \varpi_{j} t\right)}+c . c .,
$$

where $Z_{j}^{ \pm}$are the slowly varying amplitudes and

$$
\varpi_{1}=\varpi_{2}=m / 2, \quad \varpi_{3}=\Omega .
$$

Spatial and temporal symmetries constrain the equations for the evolution of $Z_{j}^{ \pm}$, as we now detail.

\section{A. Spatial symmetries}

Spatial translation symmetry acts on the TW amplitudes $Z_{j}^{ \pm}$as (cf. Eq. (4)

$$
\begin{gathered}
T_{\Theta}: Z_{j}^{ \pm} \rightarrow Z_{j}^{ \pm} e^{i \theta_{j}}, \\
\Theta=\left(\theta_{1}, \theta_{2}\right), \quad \theta_{1}, \theta_{2} \in[0,2 \pi), \quad \theta_{3}=\theta_{1}+\theta_{2} .
\end{gathered}
$$

A reflection about $\mathbf{k}_{3}$ acts as ( $c f$. Eq. (5)

$$
\kappa: Z_{1}^{ \pm} \leftrightarrow Z_{2}^{ \pm},
$$

and a rotation by $\pi$ induces ( $c f$. Eq. 6)

$$
\mathcal{R}: Z_{j}^{ \pm} \rightarrow \bar{Z}_{j}^{\mp} .
$$

We enforce equivariance under (15)-(17) to obtain the form of the TW amplitude equations to quadratic order. This truncation is sufficient to determine the leading order resonant contribution $b_{\text {res }}$ to $b\left(\theta_{\text {res }}\right)$ in (9). We have

$$
\begin{aligned}
\dot{Z}_{1}^{+}= & L_{1} Z_{1}^{+}+L_{2} Z_{1}^{-}+Q_{1} \bar{Z}_{2}^{+} Z_{3}^{+} \\
& +Q_{2} \bar{Z}_{2}^{+} Z_{3}^{-}+Q_{3} \bar{Z}_{2}^{-} Z_{3}^{+}+Q_{4} \bar{Z}_{2}^{-} Z_{3}^{-}, \\
\dot{Z}_{3}^{+}= & L_{3} Z_{3}^{+}+L_{4} Z_{3}^{-}+Q_{5} Z_{1}^{+} Z_{2}^{+} \\
& +Q_{6} Z_{1}^{+} Z_{2}^{-}+Q_{6} Z_{1}^{-} Z_{2}^{+}+Q_{7} Z_{1}^{-} Z_{2}^{-},
\end{aligned}
$$

where the remaining four equations follow from the application of (16) and (17).

We now apply a standard reduction procedure to (18) and compare this result with the SW equations (9). To 
facilitate the subsequent calculations we first introduce a phase shift to the amplitudes:

$$
Z_{1,2}^{ \pm} \rightarrow Z_{1,2}^{ \pm} e^{ \pm i \varphi / 2}
$$

where

$$
\varphi=\varphi_{2}-\varphi_{1}+\pi,
$$

with $\varphi_{1,2}$ denoting the phases of the coefficients $L_{1}$ and $L_{2}$ (i.e., $L_{1,2}=\left|L_{1,2}\right| e^{i \varphi_{1,2}}$ ). The TW equations (18) may be compactly written in the form

$$
\dot{\mathbf{Z}}=\mathbf{L Z}+\mathbf{N}(\mathbf{Z}),
$$

where $\mathbf{Z}=\left(Z_{1}^{+}, Z_{1}^{-}, Z_{2}^{+}, Z_{2}^{-}, Z_{3}^{+}, Z_{3}^{-}\right)^{T}$.

The bifurcation to SW occurs when $\left|L_{2}\right|=\left|L_{1}\right|$. As we will see in the next section, $\left|L_{2}\right| \sim\left|f_{m}\right|$, so this bifurcation condition serves to define the critical amplitude of the dominant forcing component $\left|f_{m}\right|$. The critical eigenvectors are $\mathbf{v}_{1}=(1,1,0,0,0,0)^{T}$ and $\mathbf{v}_{2}=$ $(0,0,1,1,0,0)^{T}$. We use a multi-scale perturbation calculation to accomplish the reduction to SW, writing

$$
\begin{aligned}
\mathbf{Z} & =\eta\left(A_{1} \mathbf{v}_{1}+A_{2} \mathbf{v}_{2}\right)+\eta^{2} \mathbf{Z}_{2}+\ldots, \\
\left|f_{m}\right| & =\left|f_{m}\right|^{c r i t}+\eta^{2}\left|f_{2}\right|+\ldots, \\
\frac{d}{d t} & =\eta^{2} \frac{\partial}{\partial T_{2}}+\ldots,
\end{aligned}
$$

where $\eta \ll 1$ is a small bookkeeping parameter and $A_{1,2}$ are the time-dependent SW amplitudes. At $\mathcal{O}(\eta)$ the linear problem is recovered. At $\mathcal{O}\left(\eta^{2}\right) \mathbf{Z}_{2}$ is determined. At $\mathcal{O}\left(\eta^{3}\right)$ a solvability condition yields equations for the slow variation of the $\mathrm{SW}$ amplitudes:

$$
\begin{aligned}
& \dot{A}_{1}=\Lambda_{1} A_{1}+b_{\text {res }}\left|A_{2}\right|^{2} A_{1}, \\
& \dot{A}_{2}=\Lambda_{1} A_{2}+b_{\text {res }}\left|A_{1}\right|^{2} A_{2} .
\end{aligned}
$$

The coefficients $a$ and $b_{0}$ in (9) do not appear above because the cubic order terms were omitted in (18). For the purposes of this paper, we need only point out that the "nonresonant" coefficients $a$ and $b_{0}$ are both $\mathcal{O}(\gamma)$ 12, 19, 24] (recall that $\gamma$ is a dimensionless measure of the damping). The resonant contribution is given by

$$
b_{\text {res }}=\frac{\operatorname{Re}\left\{\bar{L}_{1} S\right\}}{\operatorname{Re}\left\{L_{1}\right\}},
$$

where

$$
S=Q_{1} W e^{-i \varphi}+Q_{2} \bar{W} e^{-i \varphi}+Q_{3} W+Q_{4} \bar{W},
$$

with

$$
\begin{aligned}
W & =\left(L_{4} \bar{U}-\bar{L}_{3} U\right) /\left(\left|L_{3}\right|^{2}-\left|L_{4}\right|^{2}\right), \\
U & =e^{i \varphi} Q_{5}+2 Q_{6}+e^{-i \varphi} Q_{7},
\end{aligned}
$$

and $\varphi$ defined by (20). Our analysis applies when $\left|L_{3}\right|>$ $\left|L_{4}\right|$, i.e. when the $\mathbf{k}_{\mathbf{3}}$ mode is linearly damped.

\section{B. Temporal symmetries}

Temporal symmetries constrain the coefficients $L_{1}, \ldots, L_{4}$ and $Q_{\ell}, \ell=1, \ldots, 7$ in (18). In the absence of damping and forcing, the problem has an exact time translation symmetry

$$
T_{\Delta t}: Z_{j}^{ \pm} \rightarrow Z_{j}^{ \pm} e^{ \pm i \varpi_{j} \Delta t},
$$

with $\varpi_{j}$ given by (14), and an exact time reversal symmetry

$$
\sigma: t \rightarrow-t, \quad Z_{j}^{ \pm} \rightarrow Z_{j}^{\mp} .
$$

In the presence of finite damping and forcing, these temporal symmetries are broken. Nonetheless, they can be recast as unbroken parameter symmetries by allowing an appropriate transformation of the forcing parameters $f_{u}$ and the damping $\gamma$. With this generalization the time translation symmetry (27) becomes

$$
T_{\Delta t}: Z_{j}^{ \pm} \rightarrow Z_{j}^{ \pm} e^{ \pm i \varpi_{j} \Delta t}, \quad f_{u} \rightarrow f_{u} e^{i u \Delta t},
$$

and the time reversal symmetry (28) becomes

$$
\sigma:(t, \gamma) \rightarrow-(t, \gamma), \quad Z_{j}^{ \pm} \rightarrow Z_{j}^{\mp}, \quad f_{u} \rightarrow \bar{f}_{u} .
$$

The damping and forcing are both assumed to be small, and are of the same order, i.e., $\left|f_{u}\right| \sim \gamma \ll 1$. A Taylor expansion of the coefficients $L_{1}, \ldots, L_{4}$ and $Q_{\ell}$, consistent with (29) and (30), leads to

$$
\begin{aligned}
L_{1} & =-v_{r} \gamma, \\
L_{2} & =-i \lambda_{i} f_{m}, \\
L_{3} & =-\varrho_{r} \gamma, \\
L_{4} & =-i \mu_{i} F_{2 \Omega}, \\
Q_{\ell} & =i q_{\ell} F_{\ell},
\end{aligned}
$$

where only the leading order terms have been kept. The expansion coefficients are all real, and $v_{r}, \varrho_{r}>0$ since they correspond to damping terms. The factor of $f_{m}$ in the expansion of $L_{2}$ reflects the fact that the critical modes respond parametrically to the dominant component $f_{m}$. The factor $F_{2 \Omega}$ in the expansion of $L_{4}$ represents an analogous parametric forcing term for the damped mode composed of products of the $f_{u}$ and $\bar{f}_{u}$ whose frequencies sum to $2 \Omega$. When $2 \Omega$ forcing is present in (1), then, to lowest order, $F_{2 \Omega}=f_{2 \Omega}$; otherwise $L_{4}=0$ at $\mathcal{O}(\gamma)$.

The $F_{\ell}$ in (31e), in accordance with (29), must contain products of the $f_{u}$ (and $\bar{f}_{u}$ ) whose frequencies are such that $\left(Q_{1}, \bar{Q}_{5}\right) e^{i(\Omega-m) t},\left(Q_{2}, Q_{7}\right) e^{-i(m+\Omega) t}$, and $\left(Q_{3}, \bar{Q}_{4}, \bar{Q}_{6}\right) e^{i \Omega t}$ are time translation-invariant quantities. Since we are interested in understanding when the effect of resonant triads is significant, we focus on those cases where $b_{\text {res }}$ is $\mathcal{O}(\gamma)$ or larger; this requires that one or more of the quadratic coefficients $Q_{\ell}$ is $\mathcal{O}(\gamma)$ or larger. A straightforward calculation shows that this scaling can occur only if $\Omega \in\{m, 2 m, n, m \pm m, n-m\}, \Omega>0$, for 
some frequency $n$, and we henceforth restrict attention to these cases. Note that, since there are many frequencies in $f(\tau)$, these sets of relevant $\Omega$ values can overlap. For instance, an " $m-n$ mode" is also a " $p-m$ mode" if $n+p=2 m, n<m$. An important (and somewhat surprising) result of our symmetry calculation is that the contribution $b_{\text {res }}$ arising from a given damped mode with frequency $\Omega$ involves (at leading order) a maximum of five frequencies: the dominant frequency $m$, up to three other frequencies appearing at $\mathcal{O}(\gamma)$ in the three sets of couplings coefficients $\left(Q_{1}, \bar{Q}_{5}\right),\left(Q_{2}, Q_{7}\right)$, and $\left(Q_{3}, \bar{Q}_{4}, \bar{Q}_{6}\right)$, and potentially one more frequency, $2 \Omega$, that parametrically forces the damped mode at $\mathcal{O}(\gamma)$, thus making $L_{4}$ nonzero at leading order. The effect on the triad interaction of any additional forcing components in (11) will be $\mathcal{O}\left(\gamma^{2}\right)$ or smaller.

\section{Results}

We combine the results of Sections IIIA and IIIB to obtain leading order expressions for $b_{\text {res }}$ in (9) with explicit dependence on the damping $\gamma$, the forcing amplitudes $\left|f_{u}\right|$ and the forcing phases $\phi_{u}$. For each $\Omega$ there are a finite number of qualitatively distinct cases to consider. These are distinguished by the number of relevant frequencies involved (up to five) and the manner in which they enter the problem (through $L_{4}$ and the $Q_{\ell}$ ). Having chosen one of these, we substitute the corresponding expressions for $Q_{\ell}$, and the expansions for the remaining TW coefficients shown in (31), into equation (24) for $b_{\text {res. }}$. The results are summarized in Table $\llbracket$ and will be discussed in the next section.

To make this table of results manageable, we make use of the following definitions:

$$
\begin{aligned}
& \alpha_{1}=q_{1} q_{5}, \\
& \alpha_{2}=q_{2} q_{7}, \\
& \alpha_{3}=2 q_{6}\left(q_{3}-q_{4}\right), \\
& \alpha_{4}=q_{1} q_{7}-q_{2} q_{5}, \\
& \alpha_{5}=\left\{2 q_{1} q_{6}+q_{5}\left(q_{3}-q_{4}\right)\right\} \lambda_{i} /\left|\lambda_{i}\right|, \\
& \alpha_{6}=\left\{2 q_{2} q_{6}-q_{7}\left(q_{3}-q_{4}\right)\right\} \lambda_{i} /\left|\lambda_{i}\right|,
\end{aligned}
$$

and

$$
\begin{gathered}
P_{2 \Omega}(\Phi)=\frac{\left|L_{3}\right|+\mu_{i}\left|f_{2 \Omega}\right| \sin \Phi}{\left|L_{3}\right|^{2}-\left|\mu_{i} f_{2 \Omega}\right|^{2}} \\
R_{2 \Omega}\left(\Phi_{1}, \Phi_{2}\right)=\frac{\left|L_{3}\right| \sin \Phi_{1}+\mu_{i}\left|f_{2 \Omega}\right| \cos \Phi_{2}}{\left|L_{3}\right|^{2}-\left|\mu_{i} f_{2 \Omega}\right|^{2}} .
\end{gathered}
$$

In the above, the $q_{j}$ and $\lambda_{i}$ are defined by (31). The relevant phases $\Phi, \Phi_{1}, \Phi_{2}$ appear in Table $\square$

\section{Hamiltonian structure}

We now discuss the implications of Hamiltonian structure in the undamped problem (see 21, 26, 27, 28, 29,
30, 31]). This is a stronger assumption than that of time reversal symmetry (29) alone. We suppose, as in 12, 20, 24], that the undamped TW equations (18) can be derived from a Hamiltonian $\mathcal{H}$. Because the amplitudes $Z_{j}^{ \pm}$and $\bar{Z}_{j}^{ \pm}$need not themselves be canonically conjugate Hamiltonian variables, we write Hamilton's equations in the generalized form

$$
\dot{Z}_{j}^{ \pm}=\mp \frac{1}{r_{j}^{2}} \frac{\partial \mathcal{H}}{\partial \bar{Z}_{j}^{ \pm}}, \quad r_{1}=r_{2}, \quad r_{j} \in \mathbb{R} .
$$

This takes account of scaling transformations like $\left(Z_{1}^{ \pm}, Z_{2}^{ \pm}\right) \rightarrow r_{1}\left(Z_{1}^{ \pm}, Z_{2}^{ \pm}\right), Z_{3}^{ \pm} \rightarrow r_{3} Z_{3}^{ \pm}$that preserve the Hamiltonian character of the dynamics, and are needed to relate the underlying canonical variables to $Z_{j}^{ \pm}$and $\bar{Z}_{j}^{ \pm}$ in (18). For inviscid Faraday waves the surface height $h$ and the surface velocity potential are the underlying canonical variables (see, e.g., 27, 28]). Using this fact we find that, to leading order, $r_{1}^{2}=r_{2}^{2}=m /\left(2 k_{c}\right)$ and $r_{3}^{2}=\Omega / k_{d}$ are appropriate prefactors in (34) (see 32] where a similar factor arises in the corresponding canonical transformation).

Requiring that $\mathcal{H}$ be a real-valued function, invariant under the symmetries (15)-(17), (29) and (30), we find that the equations of motion (34) are equivalent to (18) only if $q_{1}=r q_{5}, q_{2}=r q_{7}$, and $q_{3}=q_{4}=r q_{6}$ with $r=r_{3}^{2} / r_{1}^{2}$. These conditions imply, for the results in Table Ihat

$$
\alpha_{1}>0, \quad \alpha_{2}>0, \quad \alpha_{3}=0, \quad \alpha_{4}=0 .
$$

\section{DISCUSSION}

We now discuss Table凹in some detail, highlighting the most important features of the results collected there. We then investigate the range of validity of these results, which were derived under the assumption of weak damping $\gamma$. To do this, we introduce the Zhang-Viñals Faraday wave equations and use them to perform explicit numerical calculations that demonstrate the range of $\gamma$ for which the symmetry-based results provide an accurate prediction.

\section{A. Highlights of results}

Some general comments on the organization of Table凹 are in order. Note first that there are many cases which do not need to be listed because they can be obtained simply by relabeling the different frequencies. For example, the case $(m, n, p, q, \cdot ; \Omega)=(m, n, 2 m+n, m+n, \cdot ; m+$ $n$ ) is equivalent to the case (fourth up from the bottom in Table 【) $(m, n, p, q, \cdot ; \Omega)=(m, n, m+n, n-m, \cdot ; n)$ with $n \leftrightarrow q$.

There are six groupings in the table. The first shows the five important damped modes and their contribution to $b_{\text {res }}$ when there is only one type of coupling at $\mathcal{O}(\gamma)$ or 
TABLE I: Leading resonant contribution $b_{\text {res }}$ to $b$ in (9) for the most important damped modes. For a damped mode with frequency $\Omega$, there are at most five forcing frequencies $m, n, p, q, r$ which affect $b_{\text {res }}$. Here, $m, n, p, q, r, \Omega>0$ and $x \in \mathbb{Z}^{+}$. Each expression for $(m, n, p, q, r)$, given $\Omega$, is excluded from those of entries further down the table, in which additional relationships hold. Dots indicate an arbitrary commensurate frequency, if present, which does not affect $b_{\text {res }}$ at lowest order. Entries whose listed frequencies have a common factor (e.g., those with $x$ ) are assumed to be part of a forcing function with other, relatively prime, frequencies. For $\star$ the \pm follows $\operatorname{sign}(m-n)$. See (32) and (33) for definitions of $\alpha_{1}, \ldots, \alpha_{6}, P_{2 \Omega}$, and $R_{2 \Omega}$ used below. Certain entries are reproduced from [20]; the cases that involve more than three forcing frequencies are new.

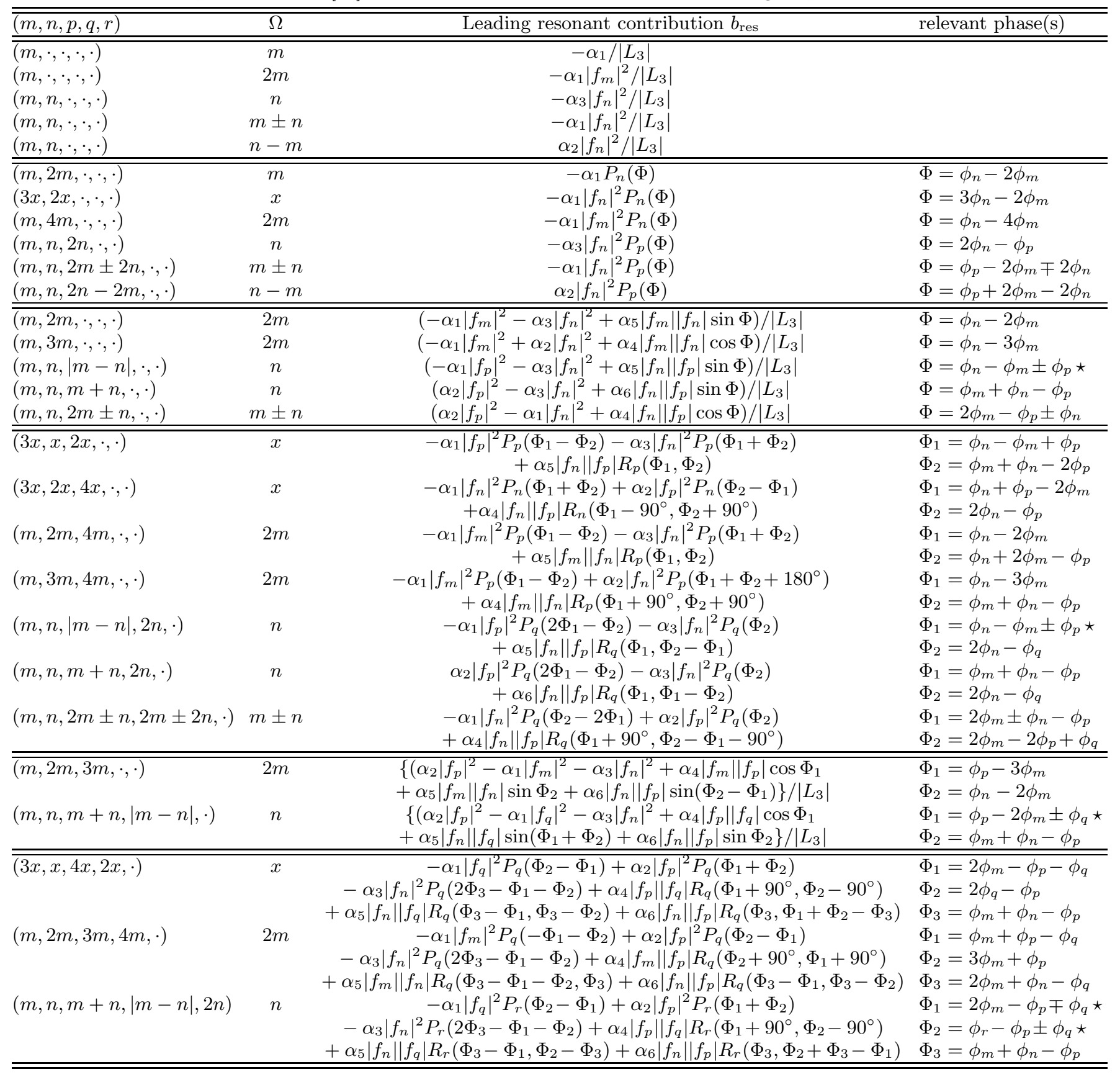

lower and no parametric forcing $f_{2 \Omega}$. In these cases there is no (leading order) dependence on the forcing phases $\phi_{u}$. In the second section the same damped modes have been parametrically forced. The factor $1 /\left|L_{3}\right|$ is then replaced by $P_{2 \Omega}(\Phi)$ of (33a). This is a strictly positive oscillatory function $\left(\left|L_{3}\right|>\left|\mu_{i} f_{2 \Omega}\right|\right.$ for damped modes) with extrema at $\Phi= \pm 90^{\circ}$. The third and fourth sections are analogous to the first and second, but with two types of coupling rather than one - similarly for the fifth and sixth sections, but with all three possible quadratic couplings (i.e., all $Q_{\ell}$ are linear in the $f_{u}$ ).

Two of the damped modes appearing in the table war- 
rant special mention. The $\Omega=m$ mode stands out because its influence is especially strong. For this mode, the largest quadratic terms in (18) are $\mathcal{O}(1)$, and the resulting contribution $b_{\text {res }}$ is $\mathcal{O}\left(\gamma^{-1}\right)$. In contrast, for all of the other damped modes, the strongest quadratic couplings take place at $\mathcal{O}(\gamma)$ and lead to $b_{\text {res }}$ of $\mathcal{O}(\gamma)$; these $\mathcal{O}(\gamma)$ contributions are of the same order as $a$ and $b_{0}$ in (9), but can still have significant effects on pattern seelction, as demonstrated in Section $\nabla$

The second special case is the $\Omega=2 m$ mode. Although this mode satisfies all the necessary temporal constraints to make a significant contribution $b_{\text {res }}$, it cannot enter into resonant triad interactions with the critical modes because its wave number is too large, i.e., $k_{d}>2 k_{c}$ and (2) cannot be satisfied; one can estimate the relevant wave numbers from the inviscid fluid dispersion relation (see [19] and Section [IVB). However, this mode may have relevance for other systems such as ferrofluids in a magnetic field where the dispersion relation is nonmonotonic [33], and hence we have kept it in the table.

A key result of Table $\llbracket$ is the important role played by the relative phases $\phi_{u}$ in the forcing function (11). For all but the most simple cases (in the first section of the table), $b_{\text {res }}$ depends on combinations of the forcing phases which are invariant under the time translation symmetry $T_{\tau}$ of (29); one phase is always arbitrary, associated with the choice of origin in time, while any physically meaningful phase must be invariant under (29). This phase dependence provides a very convenient way to tune the strength of the nonlinear interactions, as the numerical examples of Section $\nabla$ will demonstrate.

Another important aspect of Table \ pertains to the sign of $b_{\text {res }}$. Recall from the discussion of Section II that if $b_{\text {res }}>0$ interactions involving critical modes separated by the angle $\theta_{\text {res }}$ will be enhanced, whereas if $b_{\text {res }}<0$ they will be suppressed. Relations (35) mean that for simple couplings (the first two sections of Table I) the sign of $b_{\text {res }}$ is determined, and thus one knows which effect (if any) to expect. In particular, the $\Omega=m, \Omega=2 m$, and $\Omega=m \pm n$ modes are suppressing while the $\Omega=n$ mode is inconsequential. The $\Omega=n-m$ mode, in contrast, is enhancing, and thus is of great interest because it may be used directly as a selection mechanism. The effect of this difference frequency mode on pattern selection was examined in [19], and indeed, it is likely responsible for stabilizing the superlattice pattern observed in 7]. We examine the difference frequency mode further in Section $\mathrm{V}$

A final noteworthy feature of Table凹concerns the effect of parametrically forcing the damped mode with a frequency $2 \Omega$. A comparison of the factors $1 /\left|L_{3}\right|, P_{2 \Omega}(\Phi)$, and $R_{2 \Omega}\left(\Phi_{1}, \Phi_{2}\right)$ reveals the potential for a small denominator in the latter two cases. The parametric forcing can increase $\left|b_{\text {res }}\right|$ and amplify the effect of the damped mode provided this denominator does not become excessively small, which would indicate that the damped mode is nearly critical and that the reduction leading to (23) is breaking down. This feature will be exploited as well in some of the examples of Section $\nabla$

\section{B. Zhang-Viñals hydrodynamic equations}

In this subsection, we investigate the range of damping $\gamma$ for which our symmetry-based results are valid. To carry out this investigation we perform explicit numerical calculations using the Zhang-Viñals hydrodynamic equations (introduced below). In particular, we use the method described in [17] to calculate the cross-coupling coefficient $b$ in (9) as a function of $\theta$, the angle between $\mathbf{k}_{1}$ and $\mathbf{k}_{2}$ in Figure 1 It is sufficient to take $\theta \in[0,90)$ since $b\left(180^{\circ}-\theta\right)=b(\theta)$.

The Zhang-Viñals equations [21] describe the dynamics of small amplitude Faraday waves on a deep, nearly inviscid fluid layer. We use the same scaling of the equations as in [23], writing them in the form

$$
\begin{aligned}
\left(\partial_{\tau}-\gamma \nabla^{2}\right) h-\widehat{\mathcal{D}} \Phi & =\mathcal{F}(h, \Phi), \\
\left(\partial_{\tau}-\gamma \nabla^{2}\right) \Phi-\left(\Gamma_{0} \nabla^{2}-G(\tau)\right) h & =\mathcal{G}(h, \Phi)
\end{aligned}
$$

where $G(\tau)=G_{0}-f(\tau)$ and the nonlinear terms are given by

$$
\begin{aligned}
\mathcal{F}(h, \Phi)= & -\nabla \cdot(h \nabla \Phi)+\frac{1}{2} \nabla^{2}\left(h^{2} \widehat{\mathcal{D}} \Phi\right) \\
& -\widehat{\mathcal{D}}(h \widehat{\mathcal{D}} \Phi) \\
& +\widehat{\mathcal{D}}\left\{h \widehat{\mathcal{D}}(h \widehat{\mathcal{D}} \Phi)+\frac{1}{2} h^{2} \nabla^{2} \Phi\right\}, \\
\mathcal{G}(h, \Phi)= & \frac{1}{2}(\widehat{D} \Phi)^{2}-\frac{1}{2}(\nabla \Phi)^{2} \\
& -(\widehat{\mathcal{D}} \Phi)\left\{h \nabla^{2} \Phi+\widehat{\mathcal{D}}(h \widehat{\mathcal{D}} \Phi)\right\} \\
& -\frac{1}{2} \Gamma_{0} \nabla \cdot\left\{(\nabla h)(\nabla h)^{2}\right\} .
\end{aligned}
$$

Here $h(\mathbf{x}, t)$ is the fluid surface height, $\Phi(\mathbf{x}, t)$ is the surface velocity potential, and $\mathbf{x}$ is the two-dimensional spatial coordinate. The operator $\widehat{\mathcal{D}}$ multiplies each Fourier component of a field by the modulus of its wave number, i.e., $\widehat{\mathcal{D}} e^{i \mathbf{k} \cdot \mathbf{x}}=|\mathbf{k}| e^{i \mathbf{k} \cdot \mathbf{x}}$.

The equations depend on three dimensionless fluid parameters: the damping parameter $\gamma$, the gravity number $G_{0}$, and the capillarity number $\Gamma_{0}$. These fluid parameters, and the dimensionless forcing amplitudes $f_{u}$ in (11) are related to the physical parameters by

$$
\gamma \equiv \frac{2 \nu \widetilde{k}^{2}}{\omega}, \quad G_{0} \equiv \frac{g_{0} \widetilde{k}}{\omega^{2}}, \quad \Gamma_{0} \equiv \frac{\sigma \widetilde{k}^{3}}{\rho \omega^{2}}, \quad f_{u} \equiv \frac{g_{u} \widetilde{k}}{\omega^{2}} .
$$

Here $\nu$ is the kinematic viscosity, $\sigma$ is the surface tension, $\rho$ is the density, and $\omega$ and the $g_{u}$ are the Fourier amplitudes in the original (dimensioned) forcing function

$$
g(t)=\sum_{u \in \mathbb{Z}^{+}} g_{u} e^{i u \omega t}+c . c ., \quad g_{u} \in \mathbb{C} .
$$


Additionally, $\widetilde{k}$ satisfies the inviscid gravity-capillary wave dispersion relation

$$
g_{0} \widetilde{k}+\frac{\sigma \widetilde{k}^{3}}{\rho}=\left(\frac{m \omega}{2}\right)^{2},
$$

and $g_{0}$ is the usual gravitational acceleration. Note that $G_{0}$ and $\Gamma_{0}$ are not independent parameters since (38) and (40) imply that

$$
G_{0}+\Gamma_{0}=\frac{m^{2}}{4}
$$

The dimensionless dispersion relation ( $c f$. Eq. 40) also gives the natural frequency $\Omega(k)$ of undamped, unforced waves as a function of their wave number $k$ :

$$
\Omega^{2}=G_{0} k+\Gamma_{0} k^{3} .
$$

For small damping $\gamma$, (42) provides an excellent estimate of the wave number which oscillates at a given frequency, even for forced waves; we make use of this fact in Section $\nabla$ Since the critical modes oscillate with dominant frequency $m / 2$, we have $k_{c} \approx k(m / 2)=1$, where $k(\Omega)$ is the inverse of the dispersion relation from (42). One may then choose a damped mode with frequency $\Omega$, find $k(\Omega)$, and then apply (3) to estimate $\theta_{\text {res }}$.

\section{Validity of symmetry-based results}

To investigate the applicability of our results for finite values of $\gamma$, we focus on an example using three-frequency $(m, n, p)=(8,7,2)$ forcing and quantify the effect of the $\Omega=8-7=1$ damped mode; this corresponds to the penultimate entry in the second section of Table I Although this mode does not necessarily lead to the most significant resonance, we study it as an instructive example to address general questions about the validity of our symmetry results. Damped modes which play a more important role are examined in the applications in Section $\nabla$

From the Hamiltonian considerations in Section ஹwe have $\alpha_{1}>0$, and thus $b_{\text {res }}<0$. We set $\Gamma_{0}=16$ in (36), fix the ratios of the forcing amplitudes at $\left|f_{n}\right| /\left|f_{m}\right|=0.4$, $\left|f_{p}\right| /\left|f_{m}\right|=0.08$, and compute the coupling coefficient $b(\theta)$ using the method described in [17]. As predicted on the grounds of symmetry arguments, there is a dip in the plot of $b(\theta)$ around the angle $\theta_{\text {res }} \approx 23^{\circ}$ where the $\Omega=1$ mode is in spatial resonance. An example is shown in Figure 3 for $\gamma=0.1$ with $\Phi$ (which appears in the fourth column of Table (I) set to 0 .

In the discussion that follows, we study various properties of $b_{\text {res }}$ as the damping parameter $\gamma$ is varied. In this discussion, it is important to realize that the results will depend on the chosen value of $m$, on which $\gamma$ in (38) depends indirectly through (40). When generalizing the results shown below to other forcing functions it is, in fact, better to look at the quantity $\gamma / m$ ( $c f$. 38).

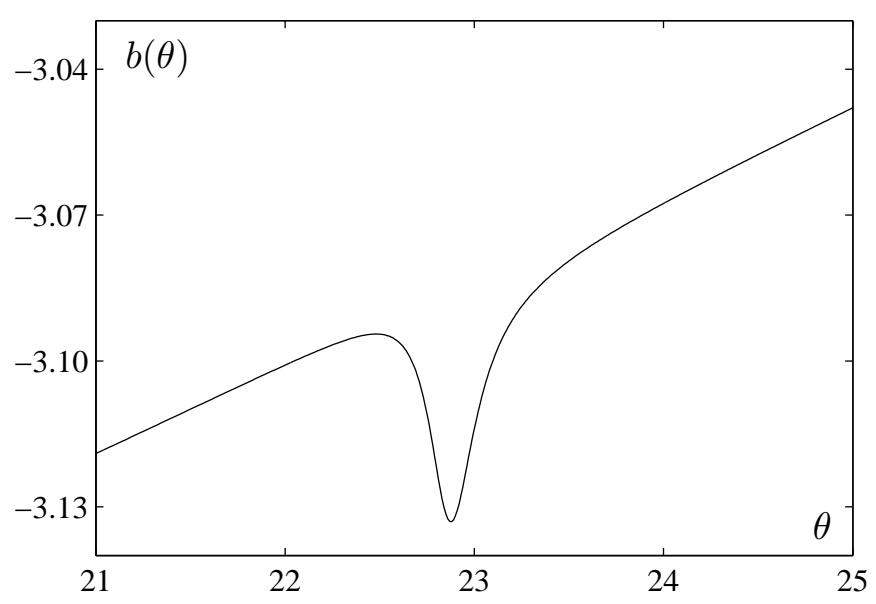

FIG. 3: Coupling coefficient $b(\theta)$ in (9) computed from (36) using three-frequency $(m, n, p)=(8,7,2)$ forcing, $\gamma=0.1$, $\Gamma_{0}=16,\left|f_{n}\right| /\left|f_{m}\right|=0.4,\left|f_{p}\right| /\left|f_{m}\right|=0.08, \phi_{m}=\phi_{n}=\phi_{p}=$ 0 .

This alternative nondimensional measure of the damping utilizes the critical wavenumber and the dominant frequency ( $m \omega$, as opposed to $\omega)$ and is therefore better suited for quantitative comparison across forcing functions with very different $m$ values. We have used the scaling (38), which utilizes the common frequency, to be consistent with previous work 19, 20, 23, 24].

We first consider the scaling of $\left|b_{\text {res }}\right|$ as $\gamma$ is varied with $\Phi=0$. It follows from the result in Table \that

$$
b_{\text {res }} \propto\left|f_{n}\right|^{2} \frac{\left|L_{3}\right|}{\left|L_{3}\right|^{2}-\left|\mu_{i}^{2} f_{p}^{2}\right|} .
$$

Furthermore, recall from (31) that $\left|L_{1,3}\right| \propto \gamma$ and $\left|L_{2}\right| \propto$ $f_{m}$. Since, at the onset of SW, $\left|L_{1}\right|=\left|L_{2}\right|$ (see Section 【II), we have $\left|f_{m}\right| \propto \gamma$. Since $\left|f_{m}\right|,\left|f_{n}\right|$, and $\left|f_{p}\right|$ are held in a constant ratio, we also have $\left|f_{n}\right|,\left|f_{p}\right| \propto \gamma$. Thus (43) becomes simply $b_{\text {res }} \propto \gamma$. This scaling is confirmed by the numerical results of Figure 4 where we hold $\Phi=0$ and plot $\left|b_{\text {res }}\right|$ as a function of $\gamma$. The numerical data is shown as points. For comparison, a line of slope one is drawn through the first data point, confirming the proportionality to $\gamma$. The theoretically predicted scaling holds reasonably well up to $\gamma \sim \mathcal{O}\left(10^{-1}\right)$, and the numerical result does not strongly diverge from the prediction until $\gamma \approx 0.5$.

Next, we examine the scaling of the half-width $\Psi$ of the dip at $\theta=\theta_{\text {res }}$. For $\theta \neq \theta_{\text {res }}$, the natural frequency of $k_{d}$ will differ from the resonant frequency $(m, 2 m, n$, etc.). At leading order, this detuning appears in the coefficient $L_{3}$ as an imaginary part, i.e., $L_{3}=-\varrho_{r} \gamma+i \varrho_{i}$ (cf. Eq. 31k). If the detuning is small, the linear approximations $\varrho_{i} \propto k_{d}-k_{\text {res }} \propto \theta-\theta_{\text {res }}$ can be used (here $k_{\text {res }}$ is the wave number associated with the resonant frequency) and so $\varrho_{i}=c\left(\theta-\theta_{\text {res }}\right)$ for some real constant $c$, i.e.,

$$
L_{3} \approx-\varrho_{r} \gamma+i c\left(\theta-\theta_{\text {res }}\right) .
$$




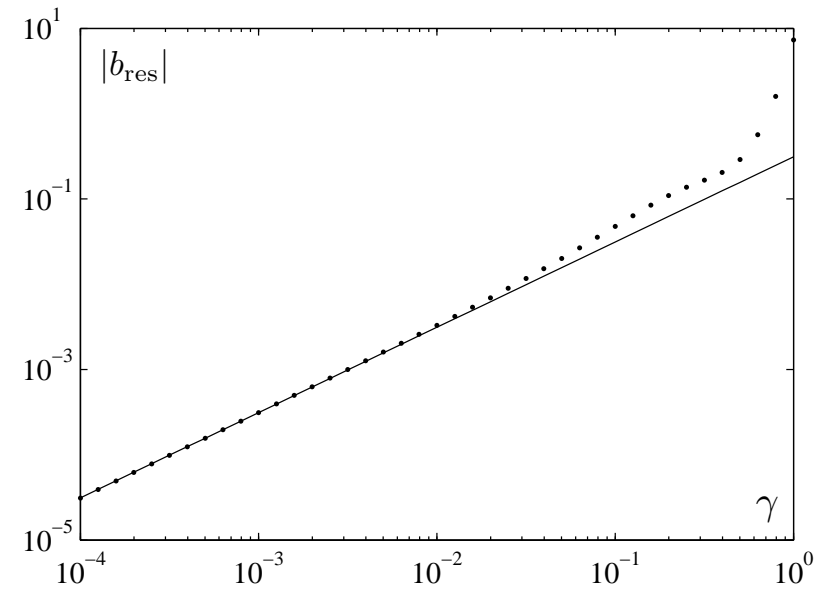

FIG. 4: Resonant contribution $b_{\text {res }}$ as a function of the damping parameter $\gamma$. The dots correspond to a numerical computation using (36). The straight line of slope one confirms the $b_{\text {res }} \propto \gamma$ scaling predicted by symmetry arguments. The capillarity and forcing parameters used are the same as those in Figure 3

Substituting this expression into the result from Table 【 shows that $\Psi \propto \gamma$. Numerical results are displayed as points on the log-log plot in Figure 5 For comparison, we plot a line of slope one fit through the first data point. As

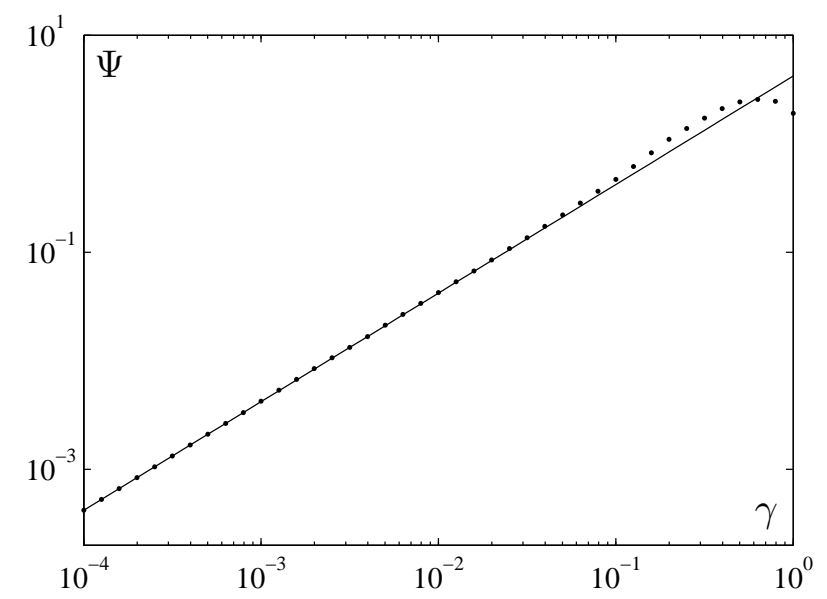

FIG. 5: Half-width $\Psi$ of the resonant "dip" as a function of the damping $\gamma$. The dots correspond to a numerical computation using (36). The straight line of slope one confirms the predicted $\Psi \propto \gamma$ scaling. The capillarity and forcing parameters used are the same as those in Figure 3

with the dip magnitude $\left|b_{\text {res }}\right|$, the theoretical prediction remains reasonable up to $\gamma \sim \mathcal{O}\left(10^{-1}\right)$.

Finally, we consider the dependence of $b\left(\theta_{\text {res }}\right)$ on $\Phi=$ $\phi_{2}-2 \phi_{8}+2 \phi_{7}$, and examine how this $\Phi$ dependence changes with increasing $\gamma$. From Table [1 we expect that the dependence is sinusoidal and, from the fact that $\mu_{i}>0$ [24] for (36), we anticipate $b\left(\theta_{\text {res }}\right)$ reach-

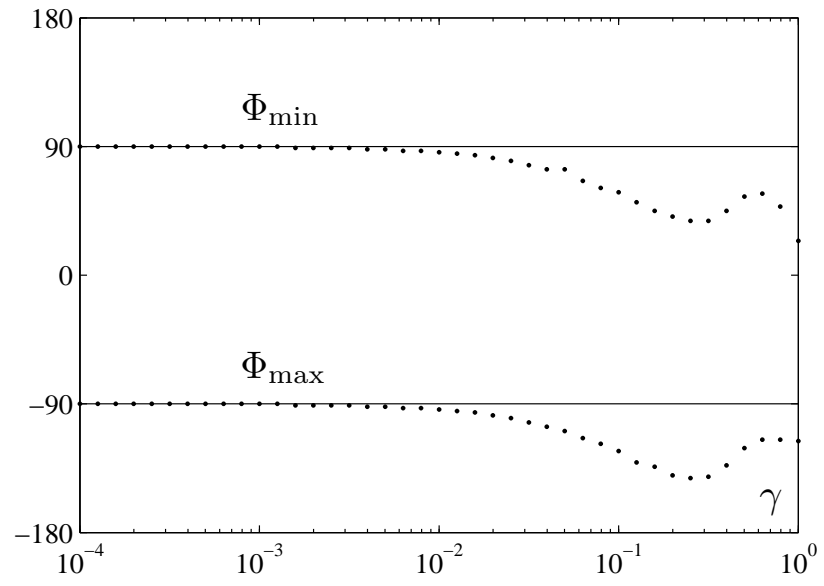

FIG. 6: The values of $\Phi$ at which $b\left(\theta_{\text {res }}\right)$ takes on its minimum and maximum values as a function of the damping $\gamma$. The dots correspond to numerical data, while the lines at $90^{\circ}$ and $-90^{\circ}$ show the predicted minimum and maximum respectively. The capillarity and forcing amplitudes used are the same as those in Figure 3

ing a maximum (i.e., having the shallowest dip) near $\Phi=\Phi_{\max }=-90^{\circ}$ and reaching a minimum (i.e., having the deepest dip) near $\Phi=\Phi_{\min }=90^{\circ}$. Figure 6 shows how the numerically calculated values (dots) of $\Phi_{\min }$ and $\Phi_{\max }$ differ from the theoretical predictions (lines) as $\gamma$ is increased. To elucidate the departure from the theoretical prediction, we show three profiles corresponding to three different values of $\gamma$ in Figure 7 In Figure $7 \mathrm{~h}$, $\gamma=0.04$ and the profile, as predicted, appears sinusoidal. The vertical lines drawn at $\pm 90^{\circ}$ agree reasonably well with the numerically calculated maximum and minimum. In Figure $7 \mathrm{~b}, \gamma=0.2$ and, although the profile is still sinusoidal, it is shifted by approximately $45^{\circ}$ with respect to the theoretical prediction. In Figure $7 \mathrm{k}, \gamma=1$, and the profile no longer resembles a sine function. This is demonstrated further by the plot in Figure $7 \mathrm{~d}$, which shows the Fourier transform of the data in Figure $\mathbf{7}$. The zero component (i.e., the $\Phi$-independent part) has been removed, and the remaining data has been normalized so that the strongest component has magnitude one. The data indicate that higher harmonics of $\Phi$ are now important. Note that the phase shift appears well before the higher harmonics come into play (see Figure $7 \mathrm{~b}$ ), a fact that can be understood as follows. The $\Phi$ dependence in Table \originates with the phase of terms in the normal form reduction, and depends on products of the coefficients in (31). If the next order terms in the expansions describing these coefficients are kept, a phase shift of $\mathcal{O}(\gamma)$ is obtained. In contrast, higher harmonics of $\Phi$ are generally associated with higher order (as opposed to next order) terms in the expansions (31). This is a result of time-translation symmetry, which requires that terms involving additional powers of the forcing amplitudes $f_{u}$ only appear in certain combinations. The specific order in $\gamma$ at which these new terms become relevant depends 

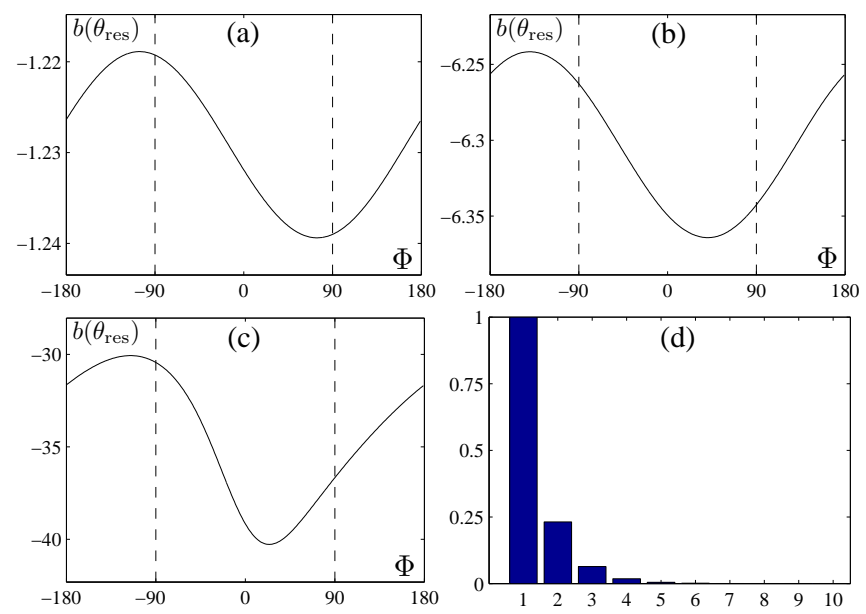

FIG. 7: $(\mathrm{a}-\mathrm{c})$ Dependence of $b\left(\theta_{\text {res }}\right)$ on the phase $\Phi$. (a) With damping $\gamma=0.04$. As predicted by the symmetry arguments in Section III the phase dependence is sinusoidal with minimum and maximum near $\pm 90^{\circ}$. (b) $\gamma=0.2$. The phase dependence is sinusoidal, but there is a phase shift of approximately $45^{\circ}$. (c) $\gamma=0.1$. The dependence is no longer sinusoidal. (d) Fourier transform of the data in (c). The zero component has been removed and the remaining data has been normalized so that the strongest component has magnitude one. The dependence on higher harmonics, e.g. $2 \Phi, 3 \Phi, 4 \Phi$ is apparent. For all plots, the capillarity and forcing amplitudes are as those in Figure 3

in complicated fashion on the particular choice of forcing frequencies.

In this section we have explored the validity of our symmetry results with respect to the small $\gamma$ assumption under which they were derived. For small $\gamma$, the symmetry results are in excellent agreement with the numerical ones. For larger $\gamma$, the scalings predicted by symmetry are not correct. However, many of the important qualitative features are preserved. In particular, even at larger $\gamma$, increasing $\gamma$ increases $\left|b_{\text {res }}\right|$. Furthermore, even though the dependence of $b_{\text {res }}$ on $\Phi$ is no longer sinusoidal, there are still special phases $\Phi_{\min }$ and $\Phi_{\max }$ which minimize and maximize $b_{\text {res }}$, suggesting that even in experiments with large damping, tuning the forcing phases may be an effective means by which to control resonant triad interactions important to pattern formation.

\section{APPLICATIONS}

The results in Table \may be used to understand - and control - certain phenomena in Faraday systems. For each of the following examples, we apply our symmetrybased methods and demonstrate the results via numerical calculations using (36).

\section{A. 1:2 temporal resonance and impulsively forced Faraday waves}

We focus on the cases for which $\Omega=m$ in Table 【 so that the the critical modes and the damped mode are in a 1:2 temporal resonance. From the Hamiltonian considerations in Section III $\alpha_{1}>0$ and thus $b_{\text {res }}<$ 0. Also, recall from Section [V] that for this case, the modes are coupled at $\mathcal{O}(1)$. Therefore, the contribution $b_{\text {res }}$ is $\mathcal{O}\left(\gamma^{-1}\right)$, which is larger than for the other cases, where $b_{\text {res }}$ is only $\mathcal{O}(\gamma)$. In short, the $\Omega=m$ mode has a very strong influence on $b(\theta)$. The implications of this well-known resonance for Faraday waves have been investigated in a number of studies, including [21].

When $f_{2 m}$ forcing is present, the size of $b_{\text {res }}$ depends on the phase $\Phi=\phi_{2 m}-2 \phi_{m}$; see the first entry in the second section of Table【 This phase dependence has previously been calculated in 14 by means of a perturbation expansion on the Zhang-Viñals model (36). Our work confirms the phase dependence in a model-independent manner, exclusively by means of symmetry considerations. The phase dependence gives us a convenient and powerful means by which to control the $1: 2$ resonance and influence the shape of $b(\theta)$. In particular, using $\Phi=90^{\circ}$ maximizes the effect of the resonance, while $\Phi=-90^{\circ}$ minimizes it.

In Figure 8 we show a numerical example for $(m, n)=$ $(1,2)$ forcing. The parameters in (36) are $\gamma=0.008$ and $\Gamma_{0}=0.125$. The forcing amplitude ratio is $\left|f_{n}\right| /\left|f_{m}\right|=$ 0.396 , which is far from the codimension-two point $\left|f_{n}\right| /\left|f_{m}\right|=3.53$ at which waves with dominant frequency $n / 2$ set in. The $\Omega=m$ mode has wave number $k(m) \approx 1.83$, and thus $\theta_{\text {res }} \approx 47.1^{\circ}$. Consistent with Table I a dip in $b(\theta)$ is found at this angle. As predicted, by choosing $\Phi=90^{\circ}$, we achieve the largest dip at $\theta_{\text {res }}$ and thus a strong suppression of patterns involving angles near this one. On the other hand, using $\Phi$ near $-90^{\circ}$, actually reduces the effect of the triad interaction by a factor $1 / 2<\left|L_{3}\right| /\left(\left|L_{3}\right|+\left|\tilde{\mu}_{i} f_{2 \Omega}\right|\right)<1$ relative to the single-frequency case, so the suppression is much weaker.

As discussed in Section III the spatiotemporal resonances we consider in this paper may also affect the selfinteraction coefficient $a$ in the one-dimensional analogue of (9), namely (12). In the case of the $1: 2$ temporal resonance, the condition $\Omega\left(k_{d}\right)=2 \Omega k_{c}$ must be satisfied along with (11). There will then be a contribution to the self-interaction coefficient $a$ in (12) whose dependence on the forcing and damping parameters is precisely that given in Table In practice, one may vary the frequency $\Omega$ by tuning the capillarity number $\Gamma_{0}$ which appears in the dispersion relation (42). In an experiment, this might be achieved by varying the base forcing frequency $\omega$ (see Eq. 38).

The results of Table \ for the 1:2 spatiotemporal resonance and its effects on the self-interaction coefficient a may be used to understand certain features of impulsively-forced Faraday waves, i.e., waves forced by a periodic sequence of impulses rather than a smooth 

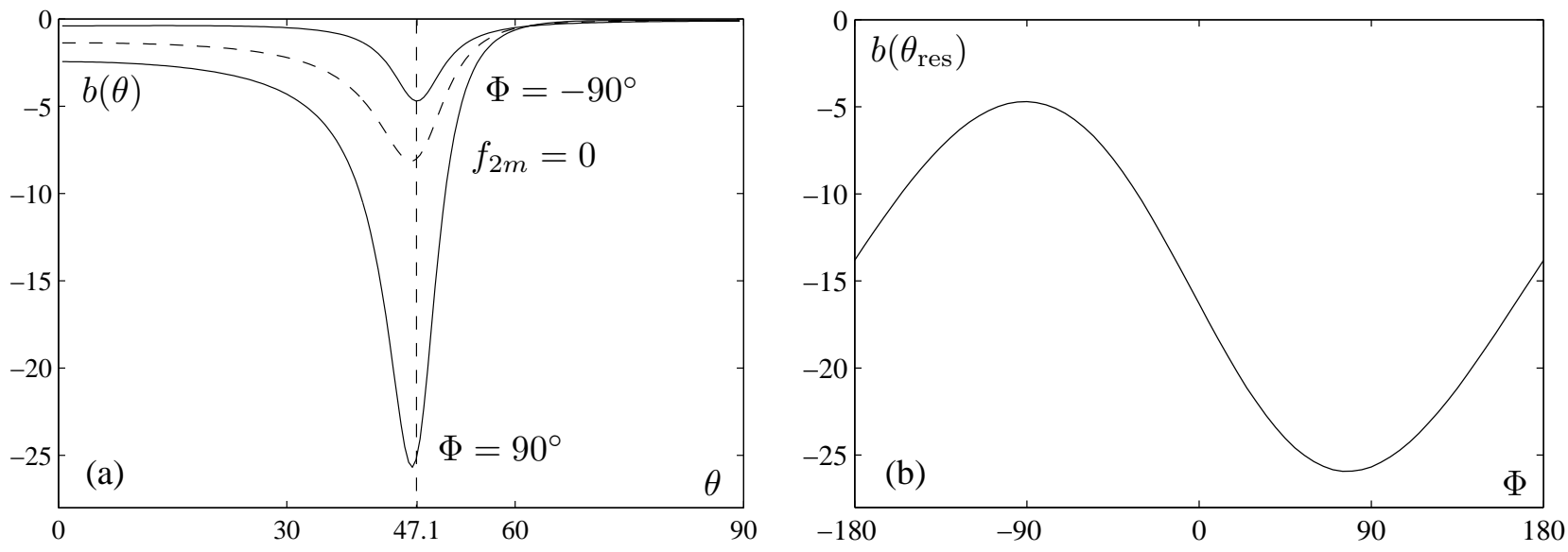

FIG. 8: Effect of relative forcing phase on the first harmonic resonance, i.e., resonance with the $\Omega=m$ mode, for $(m, n)=(1,2)$ forcing. The relevant phase $\Phi$ is given in Table @ (a) Cross-coupling coefficient $b(\theta)$ with $\Phi=90^{\circ}$ and $\Phi=-90^{\circ}$; the single frequency case (dashed line) is shown for reference. (b) Dip magnitude $b\left(\theta_{\text {res }}\right)$ versus $\Phi$. For these calculations, the parameters in (36) are $\gamma=0.008$ and $\Gamma_{0}=0.125$, and the forcing amplitude ratio is $\left|f_{n}\right| /\left|f_{m}\right|=0.396$.

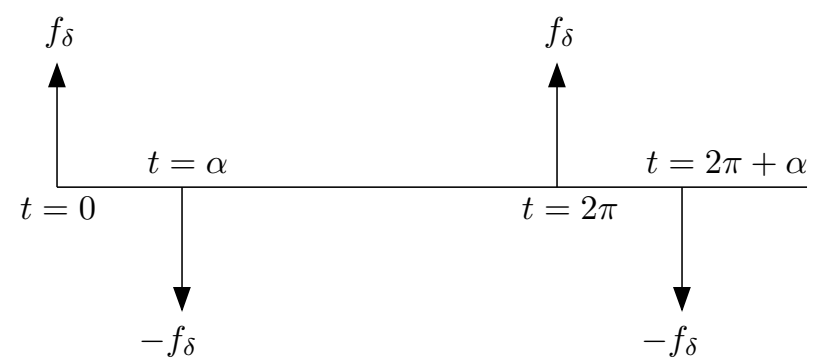

FIG. 9: Schematic representation of the asymmetric $\delta$ function forcing specified by (45).

forcing function of the form of (11). Impulsive forcing was studied first in [34] and subsequently in 22]. The original motivation for studying impulsive forcing is that it allows for a purely analytic linear stability calculation for arbitrarily large damping, in contrast to the case of smooth periodic forcing functions for which the linear analysis must be performed numerically [13, 35] and analytic approximations are only valid for small damping.

In 22 the forcing function takes the form

$$
f(t)=f_{\delta} \sum_{n=0}^{\infty} \delta(t-2 \pi n)-\delta(t-2 \pi n-\alpha)
$$

representing an alternating sequence of $\delta$-functions of strength $f_{\delta}$. The sequence has a temporal asymmetry controlled by the parameter $\alpha \in(0,2 \pi)$, which determines the amount of time between a positive pulse and the subsequent negative pulse. A depiction of (45) is shown in Figure 9, In [22], $a\left(\Gamma_{0}\right)$ is calculated from (36), and a large dip at $\Gamma_{0}=\Gamma_{\text {res }}$ is observed, where $\Gamma_{\text {res }}$ is the parameter value for which the $1: 2$ spatiotemporal resonance is satisfied. For $\gamma$ sufficiently small, it is noted that this dip becomes more negative (i.e., the corresponding $a_{\text {res }}$ becomes more negative) as the asymmetry parameter $\alpha$ is varied across the interval $(0,2 \pi)$. This observation is consistent with the results in Table as we now explain.

From Table【 there are at most two forcing frequencies which affect the $\Omega=m$ damped mode at leading order, namely $m$ and $2 m$. We therefore consider a drastic truncation of the Fourier series for the forcing function (45), keeping the first two terms, which are the only terms affecting the resonance at leading order:

$$
f(t)=f_{1} e^{i t}+f_{2} e^{2 i t}+\text { c.c. },
$$

where

$$
f_{1}=\frac{f_{\delta}}{2 \pi}\left(1-e^{-i \alpha}\right), \quad \frac{f_{\delta}}{2 \pi}\left(1-e^{-2 i \alpha}\right) .
$$

From (46), we see that varying $\alpha$ affects both the amplitudes and the phases of the forcing components. For (36) with weak damping and forcing, and for the twofrequency truncation (46), the Faraday instability occurs when $\left|f_{1}\right|=\gamma$ (this follows directly from the results in [19]). By setting $f_{\delta}$ equal to its critical value and making a translation in time, we can write the forcing function at onset as

$$
f^{c r i t}(t)=\gamma e^{i t}+F_{2} e^{2 i t}+\text { c.c. }
$$

where

$$
F_{2}=-2 i \gamma \cos \left(\frac{\alpha}{2}\right)
$$

The first entry in the second section of Table 1 indicates that the 1:2 spatiotemporal resonance produces a negative contribution $a_{\text {res }}$ to the self-interaction coefficient given by $-\alpha_{1} P_{2}(\Phi)$ where $\Phi=\arg \left(F_{2}\right)$. Using the expression (49) and simplifying reveals that

$$
a_{\mathrm{res}}=-\frac{\alpha_{1}}{\left|L_{3}\right|+2\left|\mu_{i}\right| \gamma \cos (\alpha / 2)},
$$


which decreases as $\alpha$ is increased across the interval $(0,2 \pi)$. This is consistent with the observation in 22$]$, which successfully fits numerical results to this functional form, at least for small $\gamma$. From (12) we see that the periodic striped state has a steady state amplitude of $\left|A_{1}\right|^{2}=-\lambda / a$. Thus, experimentally, the wave height may be controlled by varying $\alpha$. Larger $\alpha$ causes smaller $a$ and, consequently, larger amplitude waves.

\section{B. Stabilization of superlattice patterns with multi-frequency forcing}

We now generalize the simple one-dimensional example just presented. Our symmetry-based results suggest a methodology for "engineering" specific two-dimensional patterns through a judicious choice of forcing function. The idea is to exploit the results in Table \ in constructing a multi-frequency forcing function such that enhancing (and/or suppressing) resonances occur at carefully chosen angles. We will apply this methodology to demonstrate how a superlattice pattern of the SL-I type observed in 7] may be stabilized. Stabilization of this superlattice patterns can be related to the damped $\Omega=n-m$ "difference frequency" mode in Table 【 A demonstration is provided in 17], and further explorations are performed in [19, 20]. The method we outline below, however, results in a dramatically more pronounced stabilization than was obtained in previous work. In particular, it can lead to stable superlattice patterns at onset of the primary instability of the flat fluid surface.

Step 1: Use geometry to determine the angles for the desired enhancing (or suppressing) effects. For the SL-I pattern, the twelve dominant waves making up this pattern have wave vectors that lie at the vertices of two hexagons, one rotated by an angle $\theta_{\mathrm{h}}<30^{\circ}$ with respect to the other; see Figure [10 The stability of this pattern may be studied within the frame-

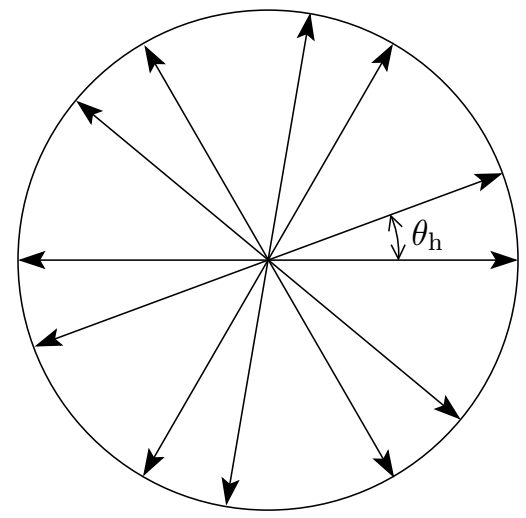

FIG. 10: Schematic of the Fourier wave vectors corresponding to the 12 dominant waves which comprise an SL-I superlattice pattern. The vectors point to the vertices of two hexagons, one rotated by an angle $\theta_{\mathrm{h}}<30^{\circ}$ with respect to the other. work of a twelve-dimensional bifurcation problem which describes the competition of these superlattice patterns with stripes, rhombic patterns, and hexagons. This approach is developed in [16, 36, 37] (the full bifurcation equations may be found there). A key result is that the stability of the superlattice pattern associated with $\theta_{h}$ depends on coefficients in the bifurcation equations which we call $\left(b_{4}, b_{5}, b_{6}\right)$, where

$$
\begin{aligned}
& b_{4}=b\left(\theta_{h}\right) /|a|, \\
& b_{5}=b\left(60^{\circ}-\theta_{h}\right) /|a|, \\
& b_{6}=b\left(60^{\circ}+\theta_{h}\right) /|a|,
\end{aligned}
$$

with $a$ and $b(\theta)$ appearing in (9). In particular, the superlattice pattern is favored when $\left(\left|b_{4}\right|,\left|b_{5}\right|,\left|b_{6}\right|\right)$ are all small. Since $b(\theta)$ may be made small in magnitude with "enhancing" resonances that cause spikes in $b(\theta)$, geometry dictates that we should arrange for such resonances to occur at one or more of the angles $\theta, 60^{\circ}-\theta, 60^{\circ}+\theta$. For a more detailed discussion, see [17].

Step 2: Use the dispersion relation and appropriate resonance conditions from Table $\square$ to find a good set of forcing frequencies which satisfy the geometrical constraints from Step 1. For our SL-I example, since we want to construct enhancing resonances, we turn our attention to the $\Omega=n-m$ "difference frequency" mode. We begin with three-frequency $(m, n, p)$ forcing, aiming to make two of $\left(\left|b_{4}\right|,\left|b_{5}\right|,\left|b_{6}\right|\right)$ small using the two difference frequency modes $\Omega=n-m$ and $\Omega=p-m$. We choose to stabilize a superlattice pattern having $\theta_{h} \simeq 20.3^{\circ}$ (this is a different SL-I pattern than the one observed in 7], but it is in the same family of patterns; see [16, 37]). The two wave numbers corresponding to the difference frequency modes satisfy the resonance conditions

$$
\begin{aligned}
& \Omega^{2}\left(k_{n-m}\right)=(n-m)^{2}, \\
& \Omega^{2}\left(k_{p-m}\right)=(p-m)^{2} .
\end{aligned}
$$

With the optimal wave numbers for these damped modes dictated by geometry, the aim is to find a set of forcing frequencies $(m, n, p)$ such that $k_{n-m}$ and $k_{p-m}$ of (52) are as close to the optimal wave numbers as possible. In practice we also vary $\Gamma_{0}$ so as to arrange for frequencies $(m, n, p)$ that are not too large - this is not strictly necessary but it eases our numerical computations to use smaller sets of integers. In this case we obtain reasonable agreement by using $(m, n, p)=(8,10,11)$ and $\Gamma_{0}=5.24$. The wave numbers predicted by (52) are $\left(k_{n-m}, k_{p-m}\right) \simeq(0.351,0.682)$ and the corresponding resonance angles of (3) are $\left(\theta_{n-m}, \theta_{p-m}\right) \simeq\left(159.8^{\circ}, 140.1^{\circ}\right)$. These will cause spikes in $b(\theta)$ at appropriately $20.2^{\circ}$ and $39.9^{\circ}$, respectively; note that the latter angle is close to $60^{\circ}-20.3^{\circ}$ (cf. Eq. 51b).

We compute the coupling coefficient from (36) with damping $\gamma=0.1$, forcing amplitude ratios $\left|f_{n}\right| /\left|f_{m}\right|=$ $1.54,\left|f_{p}\right| /\left|f_{m}\right|=1.85$, and forcing phases $\left(\phi_{m}, \phi_{n}, \phi_{p}\right)=$ $\left(0^{\circ}, 0^{\circ}, 0^{\circ}\right)$. The forcing ratios were chosen to make $b_{\text {res }}$, which is proportional to $\left|f_{n}\right|^{2}$ in one case and $\left|f_{p}\right|^{2}$ in 
the other, as large as possible, while at the same time avoiding the critical values (i.e., the modes oscillating at $n / 2$ and $p / 2$ must remain damped). The coefficients $\left(b_{4}, b_{5}, b_{6}\right)$ are represented in Figure 11], where we plot
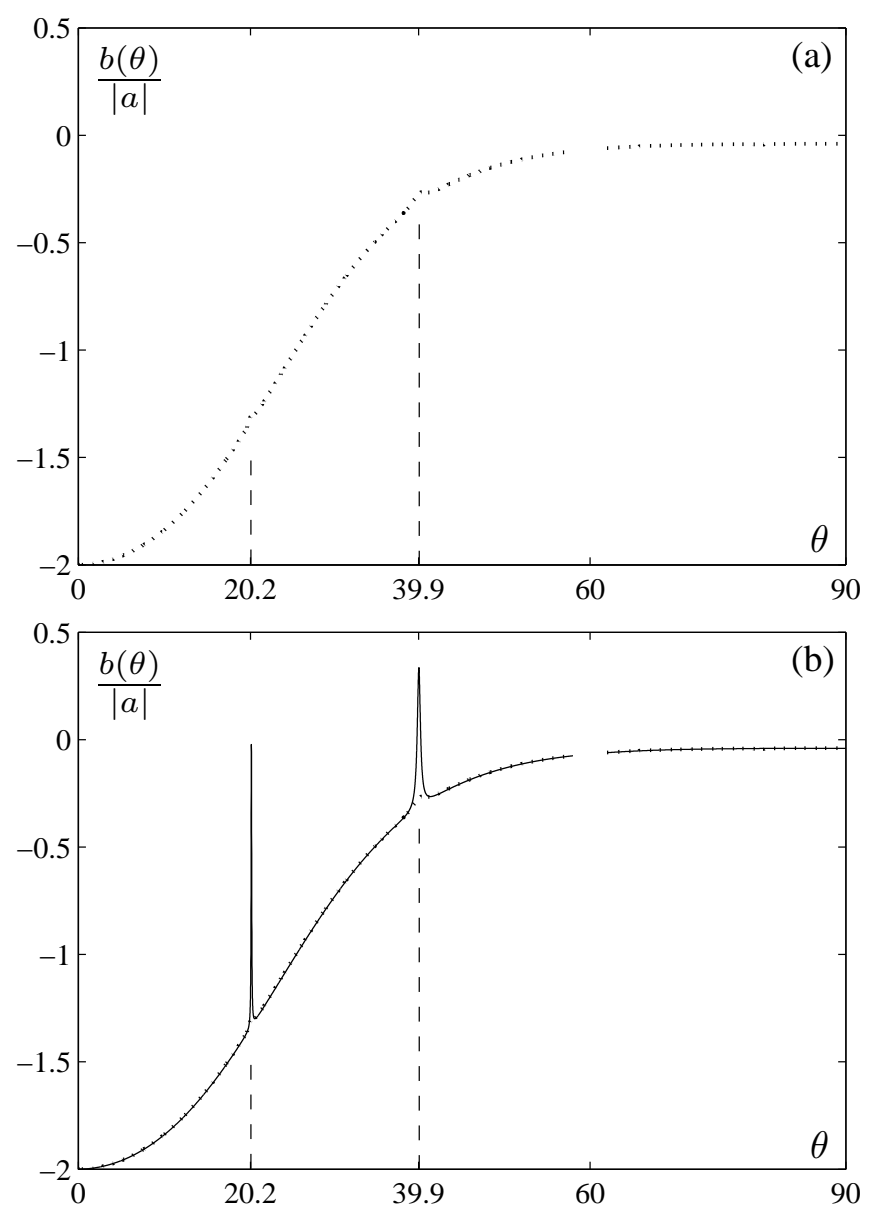

FIG. 11: (a) Coupling coefficient for computing superlattice-I pattern stability. We use three-frequency forcing with $(m, n, p)=(8,10,11)$. The two small "bumps" at $\left(\theta_{n-m}, \theta_{p-m}\right)=\left(20.3^{\circ}, 39.9^{\circ}\right)$ are due to resonance with the modes oscillating with the difference frequencies $\Omega=n-m$ and $\Omega=p-m$. No superlattice patterns are stable. (b) Like (a), but with additional forcing frequency components $(q, r)=(4,6)$ which parametrically force the difference frequency modes. The result from (a) is duplicated as a dotted line for comparison. The two bumps become two very large spikes, and the superlattice pattern with angle $\theta_{h} \simeq 20.3^{\circ}$ is stabilized. The fluid parameters used are $\gamma=0.1$ and $\Gamma_{0}=5.24$. The forcing amplitude ratios and phases used are given in the text. The region around $60^{\circ}$ corresponds to a hexagonal interaction not captured by our calculation, and thus has been removed.

$b(\theta) /|a|$ as a dotted line. As expected, there are two bumps due to the two difference frequency resonances, though they are quite small (the large dip around $\theta=0^{\circ}$ is due to resonance with the $\Omega=m$ mode). In fact, though the observed resonances at $20.3^{\circ}$ and $39.9^{\circ}$ are in excellent agreement with the prediction, the effect is far too weak to stabilize a pattern at the chosen angle, and so more work must be done.

Step 3: Use the results in Table \ to further enhance/suppress the nonlinear interactions. In this case we add the forcing components $(q, r)=(4,6)$ in order to parametrically force the damped $\Omega=2$ and $\Omega=3$ difference frequency modes and obtain larger $\left|b_{\text {res }}\right|$. In order to favor our chosen SL-I pattern, we choose $\left|f_{n}\right| /\left|f_{m}\right|$ and $\left|f_{p}\right| /\left|f_{m}\right|$ as before, and take $\left|f_{q}\right| /\left|f_{m}\right|=$ 0.184 and $\left|f_{r}\right| /\left|f_{m}\right|=0.505$. These ratios are close to (but below) their critical values when $\left|f_{m}\right|=\left|f_{m}\right|^{\text {crit }}$. We have chosen the phases to be $\left(\phi_{m}, \phi_{n}, \phi_{p}, \phi_{q}, \phi_{r}\right)=$ $\left(0^{\circ}, 0^{\circ}, 0^{\circ},-7^{\circ},-10^{\circ}\right)$. Though the arguments of Section III suggest that we should make $b_{\text {res }}$ as large and positive as possible to favor the pattern, we are working with a cubic truncation of the bifurcation equations and so we actually want $b_{\text {res }}$ such that $|b|$ is very small (as previously stated). We might have adjusted the forcing amplitude ratios to achieve this situation, but instead, we find it more convenient to vary the forcing phases away from the optimal values predicted by Table 1

The coupling coefficient appears as the solid line in Figure 11b. It nearly duplicates the result from the three-frequency case (which is included as a dotted line for comparison) but the two small bumps have become large spikes. We find that at $\theta=20.3^{\circ}$, $\left(b_{4}, b_{5}, b_{6}\right)=(0.02230,-0.01887,-0.0045)$. To study the stability of the superlattice states, we perform a bifurcation analysis using the overall forcing strength $f_{\text {tot }} \equiv \sqrt{\left|f_{m}\right|^{2}+\left|f_{n}\right|^{2}+\left|f_{p}\right|^{2}+\left|f_{q}\right|^{2}+\left|f_{r}\right|^{2}}$ as the bifurcation parameter. A branch of superlattice patterns with $\theta_{h} \simeq 20.3^{\circ}$ bifurcates transcritically from the trivial state, and the subcritical branch then turns around in a saddle-node bifurcation at a particular value $f_{\text {tot }}=f_{S N}$. At a slightly greater forcing strength $f_{S L}>f_{S N}$ (still in the subcritical regime), the superlattice pattern is stabilized, and remains stable for $f_{\text {tot }}>f_{S L}$ (at least within the realm of validity of the weakly nonlinear description provided by the bifurcation equations).

The methodology here is more successful than our previous attempts at stabilizing superlattice patterns. Our work in [17] created a spike at only one angle (as opposed to two, as here) and that in [19] did not parametrically force the damped mode and did not make an appropriate choice of phases in order to optimize the size of the spike. By combining multiple resonances with appropriately chosen phases, we have used Table $\llbracket$ to obtain dramatically increased stabilization of the desired pattern.

\section{A conjecture on quasipatterns}

The superlattice pattern discussed above belongs to one intriguing class of complex patterns; another such class is that of quasipatterns. Quasipatterns are the continuum analogues of quasicrystals. Unlike the superlattice patterns, they are not spatially periodic. However, their Fourier spectra possess discrete rotational symme- 
try. Quasipatterns have been observed in a number of Faraday wave experiments, including 6, 7, 38].

A common approach to certain types of quasipatterns has been to describe them using amplitude equations for the evolution of a number of critical modes equally spaced around a critical circle in Fourier space; see, for example, [21, 39, 40]. Recent work in 41] elucidates the technical problems with this approach. The issue is that through nonlinear interactions, the critical modes generate other modes which come arbitrarily close to the critical circle, and a center manifold reduction to a finite dimensional bifurcation problem is not possible. The usual amplitude equation description is thus without a rigorous mathematical foundation. Nonetheless, our basic physical ideas should still apply to quasipatterns. We may tune our forcing function to drive energy into modes corresponding to different resonant angles and thus favor the corresponding patterns.

For example, here we suggest a forcing function which may favor a 14-fold quasipattern, which, to date, has not been observed in Faraday wave experiments. We use the methodology outlined in the previous example. We wish to arrange for $b_{\text {res }}>0$ at the angles $\theta_{j}=j\left(180^{\circ}\right) / 14$, $j=1 \ldots 3$. This actually accounts for twelve of the angles in the pattern, since by symmetry, $b(\theta)=b\left(180^{\circ}-\right.$ $\theta)=b\left(180^{\circ}+\theta\right)$. We choose the seven-frequency forcing function $(m, n, p, q, r, s, t)=(12,17,20,27,10,16,30)$ with the capillarity parameter $\Gamma_{0}=28.8$ in (36). The $\Omega=n-m=5, \Omega=p-m=8$, and $\Omega=q-m=15$ difference frequency modes are parametrically forced by the $(r, s, t)=(10,16,30)$ components. We take forcing frequency ratios $\left|f_{n}\right| /\left|f_{m}\right|=1.2,\left|f_{p}\right| /\left|f_{m}\right|=1.6$, $\left|f_{q}\right| /\left|f_{m}\right|=2.8,\left|f_{r}\right| /\left|f_{m}\right|=0.62,\left|f_{s}\right| /\left|f_{m}\right|=1.2$ and $\left|f_{t}\right| /\left|f_{m}\right|=2.2$ and compute $b(\theta)$ from (36). The results are shown in Figure [12 The dotted line corresponds to the naive choice of zero for all of the forcing phases. The solid line corresponds to the optimized case prescribed by Table namely $\phi_{r}=\phi_{s}=\phi_{t}=90^{\circ}$. In both cases, the three difference frequency modes cause spikes in $b(\theta)$ at the required angles.

\section{CONCLUSIONS}

In this paper, we have used methods from equivariant bifurcation theory to study resonant triad interactions in Faraday waves. We have shown how the spatial and weakly broken temporal symmetries (or alternatively, parameter symmetries) may be used to determine which spatiotemporally resonant damped modes play the most important roles in pattern selection. The symmetrybased analysis not only identifies the modes, but tells us how the strength of the triad interactions depend on the frequencies, amplitudes, and relative phases of the various components in an arbitrary multi-frequency periodic forcing function. In many cases we know whether the interaction has an enhancing or suppressing effect on associated patterns. The study in this paper constitutes

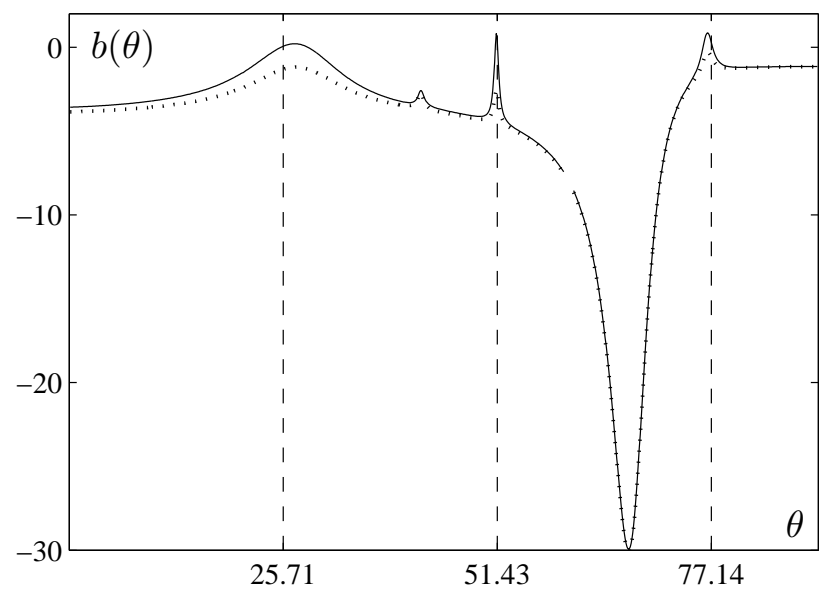

FIG. 12: Coupling coefficient $b(\theta)$ in (9) as computed from (36) for seven-frequency $(m, n, p, q, r, s, t)=$ $(12,17,20,27,10,16,30)$ forcing. The forcing phases are all 0 for the dotted line, while the solid line corresponds to the optimal choice $\phi_{r, s, t}=90^{\circ}$. The three damped modes with frequencies $n-m, p-m$, and $q-m$ are parametrically forced by the $(r, s, t)$ components. These three difference frequencies lead to spikes in $b(\theta)$ at angles which may help stabilize a 14-fold quasipattern; the desired locations of these spikes (as determined by geometry) are indicated by dashed vertical lines. The small spike around $42^{\circ}$ is due to another difference frequency resonance not of interest here. As in Figure [1] the region around $60^{\circ}$ has been removed.

a somewhat unusual situation (we know of only a few others, such as [42]) because significant information about the bifurcation coefficients, namely their scaling with respect to the physical parameters and in some cases their sign, can be obtained without resorting to calculations using the governing equations. This is possible because of the structure imposed by the parameter symmetries of the problem.

We have applied our results to impulsively forced and multi-frequency forced Faraday waves in several examples, emphasizing how the resonant interactions can be controlled by choosing judiciously the parameters in the forcing function $f(\tau)$. An appropriate choice allows one to stabilize complex patterns such as the superlattice-I pattern examined in Section $\nabla$. Techniques based on Table 1 may be useful to experimentalists wishing to observe specific patterns in the laboratory.

The results in this paper tie together many of the ideas explored in [17, 19, 20, 23] and provide an exhaustive description of the important resonant triad interactions for Faraday waves (with sufficiently weak damping). Recent experiments used multi-frequency forcing of Faraday waves in order to control the transition between different nonlinear states and to suppress spatiotemporal disorder [43]. In particular, the authors of [43] apply a perturbing third frequency to two-frequency forced patterns near a codimension-two point and interpret their results in terms of the temporal parities of the dominant 
forcing frequency and the perturbing frequency. Our results in Table \ suggest that the frequencies themselves (not just the parity) and the forcing phases are important, thus providing an alternative approach for controlling patterns.

It will be interesting to extend our work to other systems. For example, in vertically vibrated convection, Boussinesq symmetry prohibits three-wave interactions [4]. Four-wave interactions are the important nonlinear interactions, and are the building blocks of complex square superlattice patterns observed in [45, 46]. Applying techniques similar to those developed here might yield insight into this pattern selection mechanism as well.

\section{Acknowledgments}

We are grateful to Anne Catllá for a thorough reading of this manuscript and numerous helpful comments. We thank Jude Higdon for assistance with the graphical preparation of several figures. The work of CMT was supported by NSF grants DMS-9983726 and DMS9983320. The work of JP was supported by EPSRC grant GR/R52879/01. The work of MS was supported by NASA grant NAG3-2364, NSF grant DMS-0309667, and by the NSF MRSEC Program under DMR-0213745.
[1] M. Faraday, Phil. Trans. R. Soc. Lond. 121, 319 (1831).

[2] J. Miles and D. Henderson, Ann. Rev. Fluid Mech. 22, 143 (1990).

[3] H.W. Müller, R. Friedrich, and D. Papathanassiou, in Evolution of Spontaneous Structures in Dissipative Continuous Systems, edited by F. Busse and S.C. Müller (Springer, 1998), Lecture Notes in Physics, pp. 231-265.

[4] H.W. Müller, Phys. Rev. Lett. 71, 3287 (1993).

[5] W.S. Edwards and S. Fauve, Phys. Rev. E 47, R788 (1993).

[6] W.S. Edwards and S. Fauve, J. Fluid Mech. 278, 123 (1994).

[7] A. Kudrolli, B. Pier, and J.P. Gollub, Physica D 123, 99 (1998).

[8] H. Arbell and J. Fineberg, Phys. Rev. Lett. 81, 4384 (1998).

[9] H. Arbell and J. Fineberg, Phys. Rev. Lett. 84, 654 (2000).

[10] H. Arbell and J. Fineberg, Phys. Rev. Lett. 85, 756 (2000).

[11] H. Arbell and J. Fineberg, Phys. Rev. E 65, 036244.1 (2002).

[12] J. Porter and M. Silber, Phys. Rev. Lett. 89, 084501.1 (2002).

[13] T. Besson, W.S. Edwards, and L. Tuckerman, Phys. Rev. E 54, 507 (1996).

[14] W. Zhang and J. Viñals, J. Fluid Mech. 341, 225 (1997).

[15] R. Lifshitz and D.M. Petrich, Phys. Rev. Lett. 79, 1261 (1997).

[16] M. Silber and M.R.E. Proctor, Phys. Rev. Lett 81, 2450 (1998).

[17] M. Silber, C.M. Topaz, and A.C. Skeldon, Physica D 143, 205 (2000).

[18] D.P. Tse, A.M. Rucklidge, R.B. Hoyle, and M. Silber, Physica D 146, 367 (2000).

[19] C.M. Topaz and M. Silber, Physica D 172, 1 (2002).

[20] J. Porter, C.M. Topaz, and M. Silber, Phys. Rev. Lett. (in press).

[21] W. Zhang and J. Viñals, J. Fluid Mech. 336, 301 (1997).

[22] A. Catllá, J. Porter, and M. Silber (2004), in prep.
[23] M. Silber and A.C. Skeldon, Phys. Rev. E 59, 5446 (1999).

[24] J. Porter and M. Silber, Physica D 190, 93 (2004).

[25] M. Golubitsky, I. Stewart, and D.G. Schaeffer, Singularities and Groups in Bifurcation Theory: Vol. II, no. 69 in Appl. Math. Sci. Ser. (Springer-Verlag, New York, 1988).

[26] S.T. Milner, J. Fluid Mech. 225, 81 (1991).

[27] V.E. Zakharov, J. Appl. Mech. Tech. Phys. 2, 190 (1968).

[28] J.W Miles, J. Fluid Mech. 83, 153 (1977).

[29] L.J.F Broer, Appl. Sci. Res. 29, 430 (1974).

[30] P. Lyngshansen and P. Alstrom, J. Fluid Mech. 351, 301 (1997).

[31] A.C. Radder, J. Fluid Mech. 237, 435 (1992).

[32] V. P. Krasitskii, J. Fluid Mech. 272, 1 (1994).

[33] T. Mahr, A. Groisman, and I. Rehberg, J. Magn. Magn. Mater. 159, L45 (1996).

[34] J. Bechhoefer and B. Johnson, Am. J. Phys. 64, 1482 (1996).

[35] K. Kumar and L.S. Tuckerman, J. Fluid Mech. 279, 49 (1994).

[36] B. Dionne and M. Golubitsky, Z. Angew. Math. Phys. 43, 36 (1992).

[37] B. Dionne, M. Silber, and A.C. Skeldon, Nonlinearity 10, 321 (1997).

[38] B. Christiansen, P. Alstrom, and M.T. Levinsen, Phys. Rev. Lett. 68, 2157 (1992).

[39] A.C. Newell and Y. Pomeau, J. Phys. A-Math Gen. 26, L429 (1993).

[40] B. Echebarria and H. Riecke, Physica D 158, 45 (2001).

[41] A.M. Rucklidge and W.J. Rucklidge, Physica D 78, 62 (2003).

[42] P. Chossat, Dynam. Cont. Dis. Ser. A 8, 575 (2001).

[43] T. Epstein and J. Fineberg, Phys. Rev. Lett. (in press).

[44] J.L. Rogers, M.F. Schatz, J.L. Bougie, and J.B. Swift, Phys. Rev. Lett. 84, 87 (2000).

[45] J.L. Rogers, M.F. Schatz, O. Brausch, and W. Pesch, Phys. Rev. Lett. 85, 4281 (2000).

[46] J.L. Rogers, W. Pesch, and M.F. Schatz, Nonlinearity 16, C1 (2003). 